University of Tennessee Health Science Center UTHSC Digital Commons

\title{
Late Effects of lonizing Radiation on Normal Microvascular Networks
}

Vinh Nguyen

University of Tennessee Health Science Center

Follow this and additional works at: https://dc.uthsc.edu/dissertations

Part of the Therapeutics Commons

\section{Recommended Citation}

Nguyen, Vinh , "Late Effects of lonizing Radiation on Normal Microvascular Networks" (1999). Theses and Dissertations (ETD). Paper 197. http://dx.doi.org/10.21007/etd.cghs.1999.0226.

This Thesis is brought to you for free and open access by the College of Graduate Health Sciences at UTHSC Digital Commons. It has been accepted for inclusion in Theses and Dissertations (ETD) by an authorized administrator of UTHSC Digital Commons. For more information, please contact jwelch30@uthsc.edu. 


\title{
Late Effects of Ionizing Radiation on Normal Microvascular Networks
}

\begin{abstract}
Therapeutic doses of ionizing radiation result in changes in the structure and function of microvascular networks in normal tissue. Previously, we reported on the early effects of ionizing radiation on microvascular networks at 3, 7, and 30 days post-irradiation [1-3]. Data from the early time points suggested that ionizing radiation significantly alters the structure and function of microvascular networks and interferes with the normal processes of vessel maturation. Here, we present our findings on the late effects of ionizing radiation on normal tissue microvasculature at 60, 120, and 180 days post-irradiation. The cremaster muscle of Golden Syrian hamster was locally irradiated (single 10Gy dose, delivered at $2 \mathrm{~Gy} / \mathrm{min}$ ). Microvascular networks were selected in reference to a welldefined location in the tissue to reduce heterogeneity due to spatial variation. Intravital microscopy was used to measure both structural and functional parameters.

Geographic Information Systems (GIS) technology was used to establish network topology. At all late time points, the diameter of irradiated vessels was significantly larger than control. Red blood cell velocity in irradiated vessels showed a significant decrease from controls at 120 days post-irradiation and an increase at 180 days post-irradiation. Others parameters such as lineal density, tortuosity, vessel length, and vessel tone showed no significant difference between control and irradiated vessels. The hamster cremaster muscle proved to be an effective model in examining the effects of radiation on normal microvascular tissue. Together our early effect and late effect studies suggest that significant changes occur in structural and functional parameters of irradiated microvascular networks and, hence, that radiation therapy may alter the oxygen delivery capacity of normal tissue microvascular networks.
\end{abstract}

\section{Document Type}

Thesis

Degree Name

Master of Science (MS)

Program

Biomedical Engineering

Research Advisor

Mohammad F. Kiani

Keywords

ionizing radiation, microcirculation, microvascular network, late effects, blood flow

\section{Subject Categories}

Analytical, Diagnostic and Therapeutic Techniques and Equipment | Medicine and Health Sciences |

Therapeutics

\section{Comments}

One year embargo expired June 2000 


\title{
LATE EFFECTS OF IONIZING RADIATION ON NORMAL MICROVASCULAR NETWORKS
}

\author{
A Thesis \\ Presented for \\ The Graduate Studies Council \\ The University of Tennessee, Memphis
}

\author{
In Partial Fulfillment \\ Of the Requirements for the Degree \\ Master of Science \\ In the Joint Graduate Program in Biomedical Engineering \\ From the University of Tennessee \\ and \\ The University of Memphis
}

By

\section{Vinh Nguyen}

June 1999 
Copyright (C) Vinh Nguyen, 1999

All Rights Reserved 


\section{DEDICATION}

This thesis is dedicated to my parents

\section{Vi Nguyen \& Nhung Vo}

whose advice and guidance have been invaluable to me. 


\section{ACKNOWLEDGMENTS}

I would like to thank my mentor and primary advisor, Mohammad F. Kiani, Ph.D., for his guidance and patience. I would also like to thank my other committee members, James Dalton, Ph.D., and Eugene Eckstein, Ph.D. for their continued assistance throughout this study. In addition, I would like to thank Marc Sontag, Ph.D. and Sujit Ray, Ph.D. for performing the irradiation procedures, Michelle Elfervig (summer intern for 1997), Stephen Hanna (summer intern for 1988), Kai Zhao, Prabhakar Pandian, Junhong Liu and other students in the lab for their assistance in data collection. Lastly, I would like to express my thanks and gratitude to my girlfriend Ha Nguyen and my sisters Anh and Xuan-Thu who have given me direction, strength, and support throughout this endeavor. Funding for this study was provided for by the American Cancer Society (ACS), National Institutes of Health (NIH), and the School of Biomedical Engineering at The University of Tennessee, Memphis. 


\begin{abstract}
Therapeutic doses of ionizing radiation result in changes in the structure and function of microvascular networks in normal tissue. Previously, we reported on the early effects of ionizing radiation on microvascular networks at 3,7 , and 30 days post-irradiation [1-3]. Data from the early time points suggested that ionizing radiation significantly alters the structure and function of microvascular networks and interferes with the normal processes of vessel maturation. Here, we present our findings on the late effects of ionizing radiation on normal tissue microvasculature at 60,120 , and 180 days post-irradiation. The cremaster muscle of Golden Syrian hamster was locally irradiated (single 10Gy dose, delivered at $2 \mathrm{~Gy} / \mathrm{min}$ ). Microvascular networks were selected in reference to a well-defined location in the tissue to reduce heterogeneity due to spatial variation. Intravital microscopy was used to measure both structural and functional parameters. Geographic Information Systems (GIS) technology was used to establish network topology. At all late time points, the diameter of irradiated vessels was significantly larger than control. Red blood cell velocity in irradiated vessels showed a significant decrease from controls at 120 days post-irradiation and an increase at 180 days post-irradiation. Others parameters such as lineal density, tortuosity, vessel length, and vessel tone showed no significant difference between control and irradiated vessels. The hamster cremaster muscle proved to be an effective model in examining the effects of radiation on normal microvascular tissue. Together our early effect and late effect studies suggest that significant changes occur in structural and functional parameters of irradiated microvascular networks and, hence, that radiation therapy may alter the oxygen delivery capacity of normal tissue microvascular networks.
\end{abstract}




\section{Table of Contents}

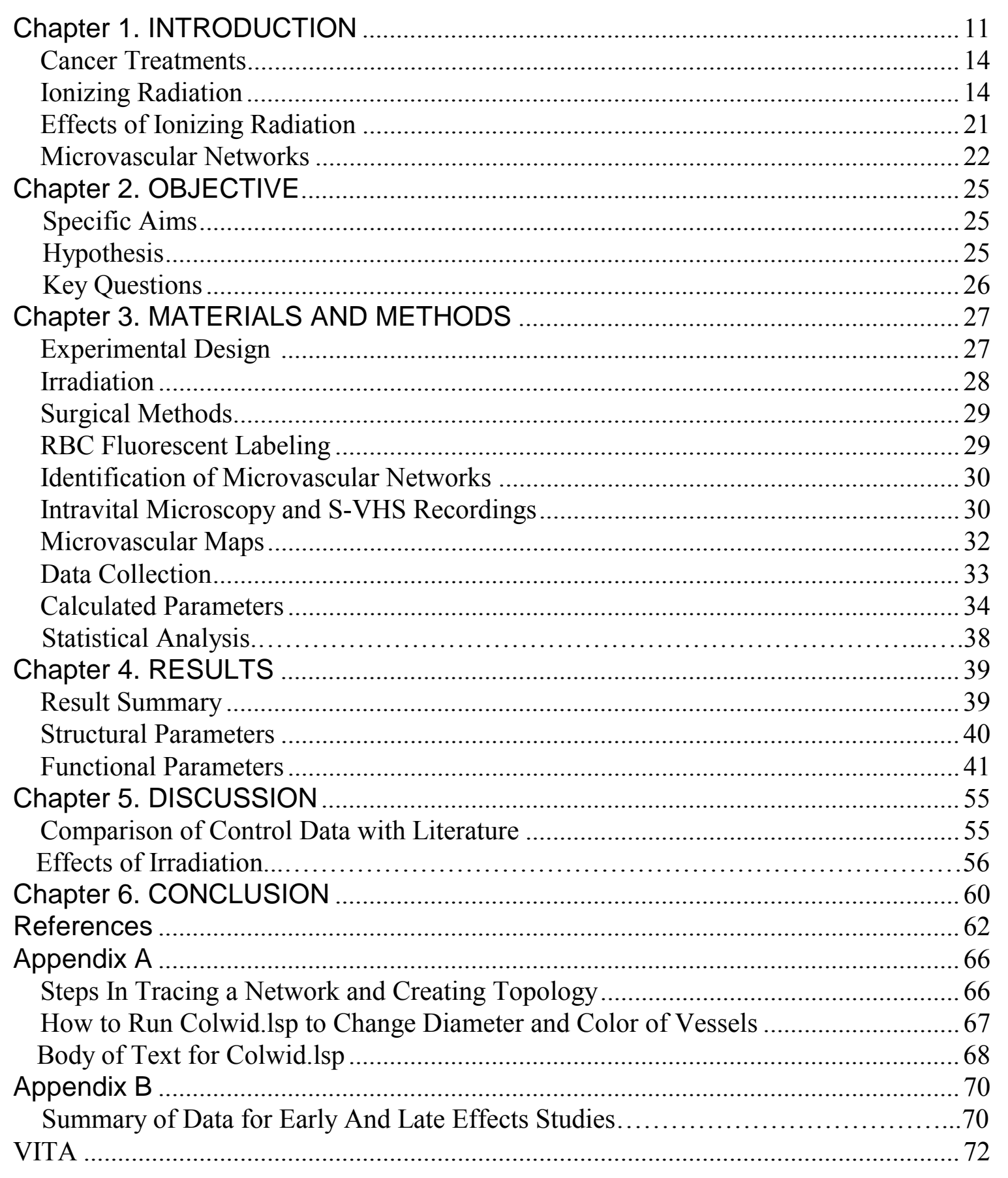




\section{LIST OF TABLES}

Table 4-1. The Interactions of Time Post-Irradiation and Radiation Status for All Data

Table 4-2. The Interactions of Vessel Type and Radiation Status for 60 Days PostIrradiation.............................................................................49

Table 4-3. The Interactions of Vessel Type and Radiation Status for 120 Days PostIrradiation.

Table 4-4. The Interactions of Vessel Type and Radiation Status for 180 Days PostIrradiation.

Table 4-5. The Interactions of Time Post-Irradiation and Radiation Status for Type 1

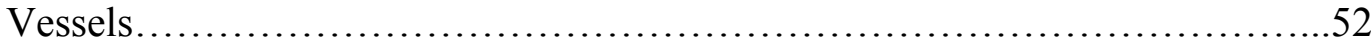

Table 4-6. The Interactions of Time Post-Irradiation and Radiation Status for Type 2

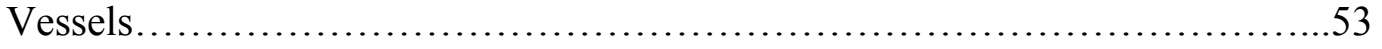

Table 4-7. The Interactions of Time Post-Irradiation and Radiation Status for Type 3

Vessels..............................................................................54 


\section{LIST OF FIGURES}

Figure 1-1. Compton effect. From Hendrickson et al. [4] ............................15

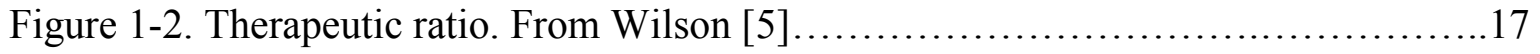

Figure 1-3. Different therapeutic ratios exist in different clinical circumstances depending on the radiosensitivity (dose response curves) for tumor versus the critical normal tissue in the treatment field. From Rubin et al. [6]................ 18

Figure 1-4. A sigmoidal dose-response curve characterizes the relation between both tumor control and normal tissue damage versus dose. From Rubin et al. [6].......18

Figure 1-5. A clonogenic cell survival curve represents the fraction or proportion of

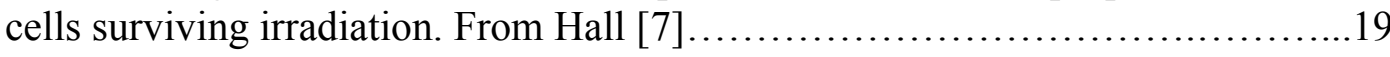

Figure 1-6. Cell repair and fractionation effect. From Elkind et al.[8] ...................20

Figure 3-1. Experimental design used to study the late effects of ionizing radiation exposure of normal tissue microvascular. Adapted from Roth $[1] \ldots \ldots \ldots \ldots \ldots \ldots . .28$

Figure 3-2. Block diagram of video microscopy system. From Roth $[1] \ldots \ldots \ldots \ldots \ldots \ldots . \ldots . \ldots$.

Figure 4-1. Animal weight at 60, 120, 180 days post-irradiation $(\mathrm{N}=5$ animals per time point per treatment group)...

Figure 4-2. Vessel diameter ( $\mathrm{N}=5$ animals per treatment group per time point) of type $1,2,3$, and all vessels at 60,120 , and 180 days post-irradiation.

Figure 4-3. RBC velocity at $60,120,180$ days post-irradiation $(\mathrm{N}=5$ animals per time point per treatment group)...

Figure 4-4. Vessel hematocrit at 60, 120, 180 days post-irradiation $(\mathrm{N}=5$ animals per time point per treatment group).

Figure 4-5. Vessel hematocrit $(\mathrm{N}=5$ animals per treatment group per time point) of type 1,2,3, and all vessels at 60,120 , and 180 days post-irradiation

Figure 4-6. The product of vessel hematocrit and $\mathrm{RBC}$ velocity $(\mathrm{N}=5$ animals per treatment group per time point) of type 1,2,3, and all vessels at 60, 120, and 180 days post-irradiation.............................................45

Figure 4-7. Vessel wall shear stress ( $\mathrm{N}=5$ animals per treatment group per time point) of type $1,2,3$, and all vessels at 60,120 , and 180 days post-irradiation............46 


\section{LIST OF SYMBOLS}

Animal Weight

Discharge Hematocrit

Flow Rate

RBC Flux of Daughter Vessel

RBC Flux of Parent Vessel

Lineal Density

Microvascular Network Surface Area

Number of Animals

Number of Vessels

Red Blood Cell Flux

Red Blood Cell Transit Time

Red Blood Cell Velocity

Red Blood Cell Velocity * Hematocrit

Tortuosity

Arteriolar Tone

Vessel Diameter

Vessel Length

Vessel Hematocrit

Vessel wall shear rate

Vessel wall shear stress

Adapted from Roth [1].
W (g)

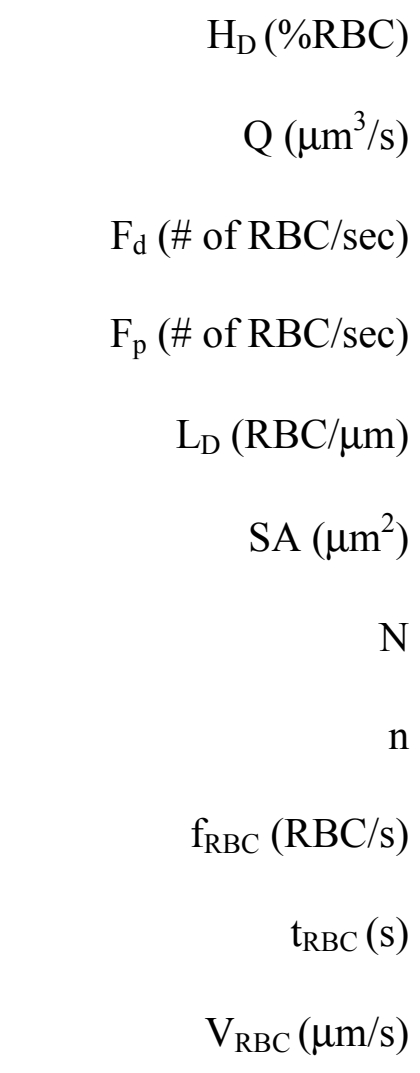

$\mathrm{V}_{\mathrm{RBC}} \mathrm{H}_{\mathrm{t}}(\% \mathrm{RBC} \mu \mathrm{m} / \mathrm{s})$

$\mathrm{V}_{\text {tone }}(\%$ change $)$

$\mathrm{d}(\mu \mathrm{m})$

$\mathrm{L}(\mu \mathrm{m})$

$\mathrm{H}_{\mathrm{t}}(\% \mathrm{RBC})$

$\gamma(1 / s)$

$\tau_{\mathrm{W}}\left(\mathrm{N} / \mathrm{m}^{2}\right)$ 


\section{LIST OF ABBREVIATIONS}

Analysis of Variance

ANOVA

Control

$\mathrm{CN}$

Entrance Probability

EP

Geographic Information Systems

GIS

Input / Output

$\mathrm{I} / \mathrm{O}$

Intraperitoneal

IP

Intravenous

IV

Irradiated

IR

Red Blood Cell

$\mathrm{RBC}$

Super VHS

SVHS

Travel Time

TR

Vessel Identification

ID

Vessel Surface Area

VSA

White Blood Cell

WBC

From Roth [1]. 


\section{Chapter 1. INTRODUCTION}

Cancer is the second leading cause of death in the United States and in many other industrialized countries. However, this dreaded disease, which was synonymous with death, has yielded to the advances in modern treatments that include surgery, radiation therapy, and chemotherapy. Cancer cells can arise in any body tissue, at any age. Characteristically, they invade local tissues by direct extensions, or they spread throughout the body through lymphatic or vascular channels. As infectious diseases are brought under control and increased longevity results, the incidence of cancer in a given population will certainly rise. In this country, one in every four Americans will be diagnosed with cancer, and approximately 50\% to $60 \%$ of those diagnosed as cancer patients will probably die from their disease [9].

The exact cause of cancer remains undetermined. Although there are readily recognizable histopathologic differences between the cancer cell and the normal cell, few metabolic differences have been determined. The biochemical structure of nucleic acids is similar in both types of cells, but some reduction of vital proteins, such as respiratory enzymes, cytochromes, and cytochrome oxidase exists in cancer cells [10]. Evidence suggests the conversion of normal cellular genes (c-onc) responsible for cellular metabolism, division, and maturation, to malignant oncogenes leading to uncontrolled growth [10]. More than one of these genes could be involved, and a set of anti-oncogenes (anti-onc) was recently identified that function as cellular repressors. This network of coordinated gene expression is ultimately responsible for the activation of uncontrolled cell growth producing a malignancy [12].

Of the known carcinogenic agents- chemicals, viruses, and radiation- chemicals have emerged as the most important in the induction of human cancers. Their common feature is that they are electrophilic reactants, arising through metabolism [10]. Environmental and industrial 
carcinogens such as tobacco and alcohol have been linked to cause lung cancer and upper aerodigestive tract malignancies. Radiation exposure is high on the list of carcinogens because of its known destructive action. However, a small fraction-less than $1 \%$ - of all cancer is attributable to this cause. Drug-induced cancers are also of particular concern to the oncologists because a new tumor can arise after curing the first. For example, the use of alkylators for treatment of cancer (e.g. melphalan and cyclophosphamide) is known to induce other leukemias and bladder cancers [13]. In addition, the combination of multi-agent chemotherapy and irradiation, used in treating a variety of cancers, has also resulted in secondary leukemias. Finally, a few type DNA and RNA viruses have been shown to cause human malignancies. The Epstein-Barr virus and hepatitis B virus are two agents that chronically infect humans and are associated with neoplasms.

Almost by definition, both benign and malignant cells must proliferate in order to produce a tumor (i.e., a mass). Tumor cell proliferation is autonomous, and thus, its control mechanism must therefore be abnormal [14]. However, malignant cells may respond to normal control signals (e.g., proliferation of breast cancer cells may be stimulated by low levels of estrogen and inhibited by high levels). The growth of some tumor cells appears to depend upon growth factors that the tumor cells themselves secrete, inappropriately. In normal proliferating tissues such as the intestinal crypt cells, the cell cycle is generally $24-48$ hours. Likewise, malignant cells have similar cell cycle time. However, the abnormal growth of a tumor's mass is due to the persistence of cycling rather than a rapid rate of cycling. In normal proliferating tissues, stem cells divide and daughter cells begin to differentiate. After a defined number of divisions, the daughter cells differentiate to terminal cells and cannot divide again. In tumor arising in stem cell systems, proliferation per se is not abnormal because the normal cell of origin 
proliferates. Instead, differentiation to form terminal cells is either abnormal and delayed or absent in cells of the malignant clone [14]. In tumors arising from cells that are normally nonproliferating, such as liver cells, the activation of proliferation is a recognized abnormality, and proliferative signals are presumed to be altered.

As for tumor growth rate, when tumors are small, the number of cells in the tumor increases exponentially and rapidly as would be predicted from the cell cycle time of 1-2 days. However, as the tumor enlarges, the number of cells in the tumor ceases to grow exponentially but instead grows progressively more slowly. More and more tumor cells cease to proliferate and more and more tumor cells die. One reason for this is that as the mass grows, many cells become distant from the blood vessels and therefore become nutrient-deprived; they stop proliferating and often die (necrose). Most tumors have areas of necrosis. In addition, in many tumors, a proportion of the cells appear to differentiate to terminal cells. This must be the case because if a 10 gram tumor doubled every 2 days, as an individual cell can, it would weigh over $1 \mathrm{~kg}$ in 2 weeks, and over $100 \mathrm{~kg}$ in another 2 weeks. Finally, it is also hypothesized that some cells accumulate so much genetic damage that they die [14].

Benign tumor cells, which also can proliferate autonomously, can be distinguished from malignant cells by the characteristic that malignant cells invade neighboring tissues or metastasize to distant regions. Malignant tumor can invade into blood or lymph vessels and metastasize to other parts of the body. The regulation of this program is not understood, but invasiveness is a normal characteristic of macrophages, lymphocytes, granulocytes, fibroblasts in wound, certain migrating embryonal cells, and placental cells, for instance. 


\section{Cancer Treatments}

Treating cancer is difficult because it is not a single disease and because all the cells in a single tumor population do not behave in the same way [15]. Many believe that cancers are derived from a single abnormal cell, but by the time a tumor is detected, most cancers contain diverse populations of cells. Some of the cells may metastasize and others do not. Some may divide and others may not. Because of this diversity, a single chemotherapeutic drug may destroy susceptible populations of cells but permit resistant populations to proliferate. This is a big drawback of chemotherapy. Certain tumor cells may be simultaneously resistant to completely unrelated drugs, a phenomem called multi-drug resistance [15]. This broad-based resistance to chemotherapeutic drugs is due to a plasma membrane protein called P-glycoprotein (PGP) that accumulates in resistant tumor cells. PGP actively transports drugs out, and the cells are not killed [15]. Another obstacle encountered by blood-borne anticancer drugs is the physical barrier posed by solid tumors such as those that arise in the breast, colon and other organs. Because of their size, deep within such tumors are high-pressure areas that cause blood vessels to collapse [16;17]. This makes it difficult, if not impossible, for blood-borne anticancer drugs to penetrate the tumor. For these reasons, chemotherapy is often used in combination with others method of treatment (e.g., radiation therapy, surgery, etc.).

\section{Ionizing Radiation}

Ionizing radiation from radium was discovered by Roentgen and Curie in the late 1890s. Since then ionizing radiation has been used to treat a variety of diseases, particularly cancer. Today, radiation therapy is the second most commonly used method of treatment for cancer after 
surgery. Of the 1.5 million diagnosed cancer cases per year in the U.S., around 400,000 patients undergo some form of radiation therapy [18].

Ionizing radiation can be classified into two types: electromagnetic or particulate.

Electromagnetic radiation consists of $\mathrm{x}$-rays and gamma rays. X-rays are produced in a machine that accelerates electrons to high energy and then stops them using tungsten or copper. Part of the stopping energy is dissipated as heat and the remainder is converted into x-rays. On the other hand, gamma rays are emitted from the nucleus of radioactive isotopes through unstable nucleus decay. Particulate radiation includes electrons, protons, neutrons, and alpha particles. Electrons are negatively charged particles that can be accelerated in a variety of machines. Protons can also be accelerated because they possess a positive charge. Neutrons are produced by colliding protons or deuterium into appropriate targets. Alpha particles currently are of no particular clinical importance.

X-rays and gamma rays are absorbed in tissue by a process called the Compton effect. The process involves a photon interacting with an orbital electron, overcoming the electron binding energy and imparting some kinetic energy that ejects the electron in one direction while the energy-reduced photon scatters in a different direction (see Figure 1-1).

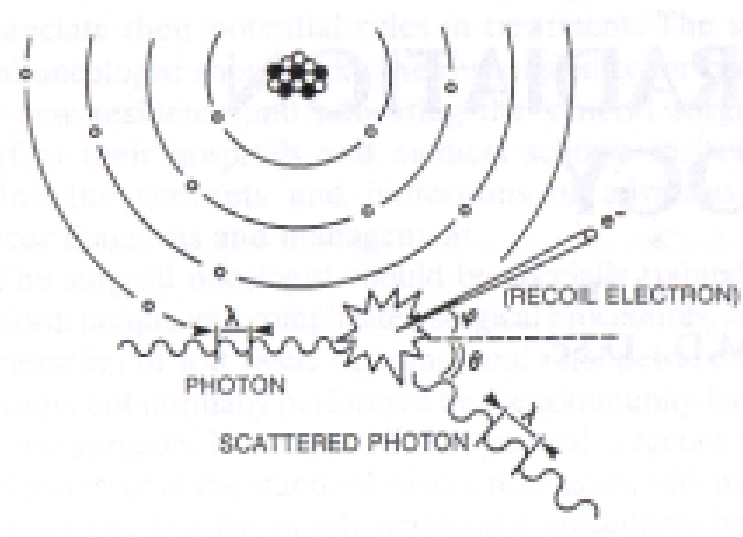

Figure 1-1. Compton effect. An energetic photon interacts with an orbital electron, overcoming the electron binding and imparting some kinetic energy that ejects the electron in one direction while the energy-reduced photon scatters in a different direction. From Hendrickson et al. [4]. 
The recoil electron will interact with other electrons and gradually lose its kinetic energy. The scattered photon may react further and give up more energy as it traverses the tissues. On the other hand, protons and neutrons interact directly with the molecular nucleus, ejecting varying lower-energy nuclear fragments. Because protons and neutrons are heavier particles, the energy deposits are closer together than those produced by photons and electrons. This deposition is called high linear energy transfer (LET) radiation and is different from the low-LET radiation of photons and electrons. Conceptually, low-LET radiation is like skipping stones on water. A light stone (photon or electron) thrown with moderate energy will touch the water and skip each time with less distance until all the energy has been dissipated. High-LET radiations would be heavier stones requiring greater energy behind the throw, but as it skips, it impart a great deal of energy to the water but with much less distance between each skip [4].

Ionizing radiation can be delivered in two ways: teletherapy and brachytherapy. In teletherapy, the source of the radiation is some distance from the patient. This long distance is advantageous because it allows room for a uniform dosage to be delivered using modifying devices (i.e., collimator) placed between the source and the patient. In brachytherapy, the radioactive sources (e.g., radium) are placed directly into the tumor site. Because of the inverse square law, the radiation dosage is high near the sources in the tumor and much lower in the normal tissues further away from the radioactive sources. A combination of teletherapy and brachtherapy is used in the treatment of many common tumors [4].

Optimal radiation therapy of malignant disease is achieved when the tumor is completely eradicated and the surrounding normal tissues show little evidence of structural and functional damage. Although this "ideal effect" is being obtained more frequently, one accepts a certain degree of permanent residual damage as a sequel to the destruction of a lethal tumor [6]. This 
ability to eradicate the tumor without undue complication or destruction of normal tissues is the essential factor in tumor radiocurability and is referred to as the therapeutic ratio [6]. The relation between radiation dose and the probability of control of a homogeneous group of tumors is sigmoidal; that is , with increasing radiation doses, more and more neoplastic cells are killed, until ultimately all clonogenic cells are destroyed and a cure is achieved. The same principle of radiation killing, however, also applies to normal cells and tissues, and so the probability of normal tissue complications and tumor cure are similar. Because there are vast varieties of human tumors and different normal organs and tissues, each with different cell types, the overlap in the tolerance dose of normal tissues and the tumor dose for cure is inevitable. It is this relation between normal tissue tolerance and tumor lethal dose that determines the therapeutic ratio (see Figure 1-2) [5].

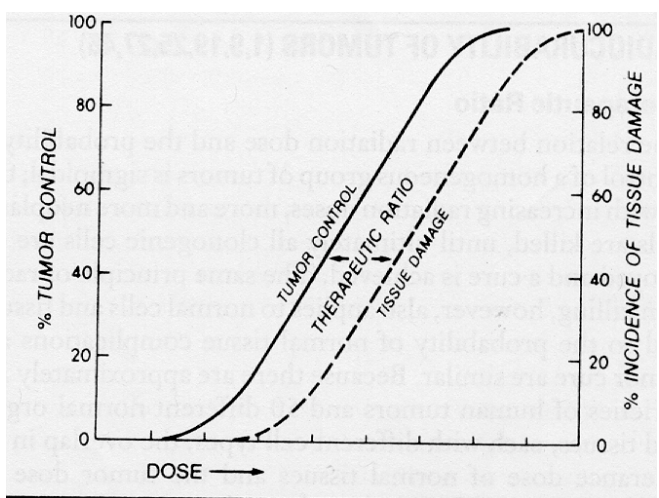

Figure 1-2. Therapeutic ratio. The response curves of both tumor cure and normal tissue complication are sigmoidal. The differential in dose to achieve these effects is the therapeutic ratio. From Wilson [5].

The therapeutic ratio is favorable when the tumor curve is to the left of the normal tissue dose effect curve (Figure 1-3a). This means the tumor can be destroyed without excessive normal tissue complication. When the two curves are identical (Figure 1-3b), the therapeutic ratio is less favorable, but may be improved by precise radiation treatment, so that a greater dose is delivered to the tumor than to the normal surrounding tissue [6]. Finally, if the tumor ablation curve is to the right of the vital normal tissue (Figure 1-3c), the circumstance is most 

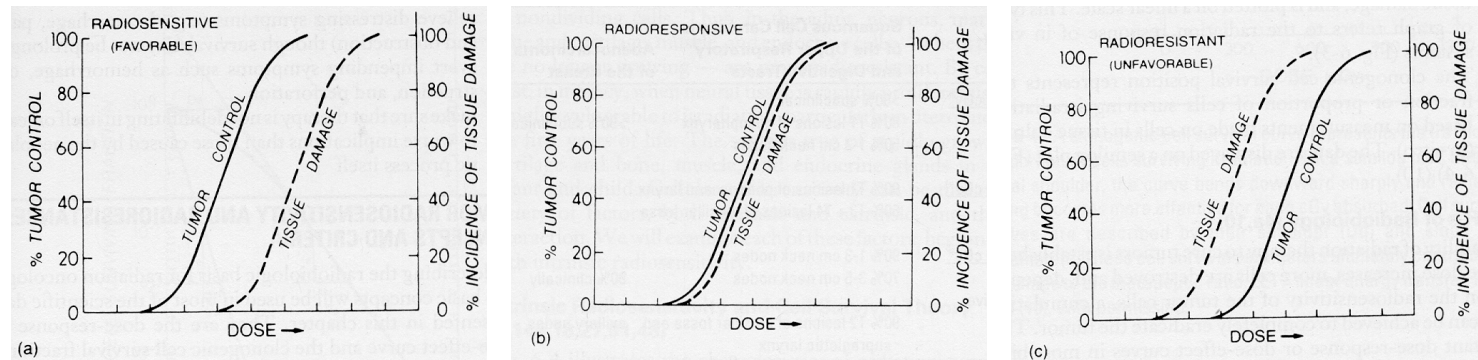

Figure 1-3. Different therapeutic ratios exist in different clinical circumstances depending on the radiosensitivity (dose response curves) for tumor versus the critical normal tissue in the treatment field. These figures show (a) a favorable ratio, (b) a less favorable ratio, and (c) an unfavorable ratio. From Rubin et al. [6].

unfavorable. To reverse this situation, innovations and combined modality approaches (e.g., radiation therapy with chemotherapy) are essential to a good outcome [6].

In describing the radiobiologic basis for radiation oncology, two concepts are often used.

They are the dose-response curve and the clonogenic cell survival fraction. The dose-response curve (see Figure 1-4) is sigmoidal and characterizes the relationship between dose and a response on effect, such as tumor control or normal tissue damage [6]. As the dose increases, more cells are destroyed, and depending on the radiosensitivity of the tumor cells, a cumulative dose can be achieved to completely eradicate the tumor.

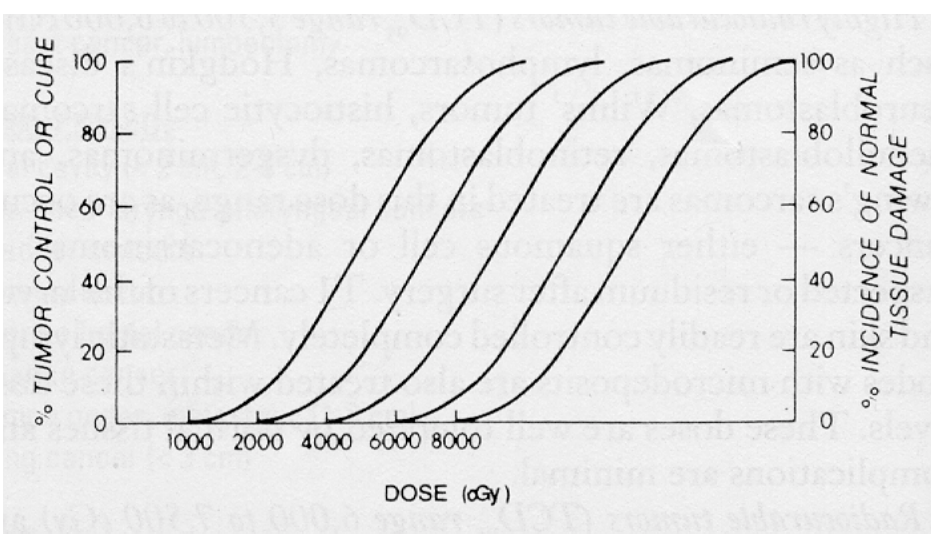

Figure 1-4. A sigmoidal doseresponse curve characterizes the relation between both tumor control and normal tissue damage versus dose. As the radiation dose increases, a threshold is reached beyond which both tumor control and normal tissue injury increase. From Rubin et al. [6]. 
The clonogenic cell survival graph (see Figure 1-5) represents the fraction or proportion of cells surviving irradiation based on measurements made on cells in tissue culture. Following an initial shoulder region, the radiation killing is essentially

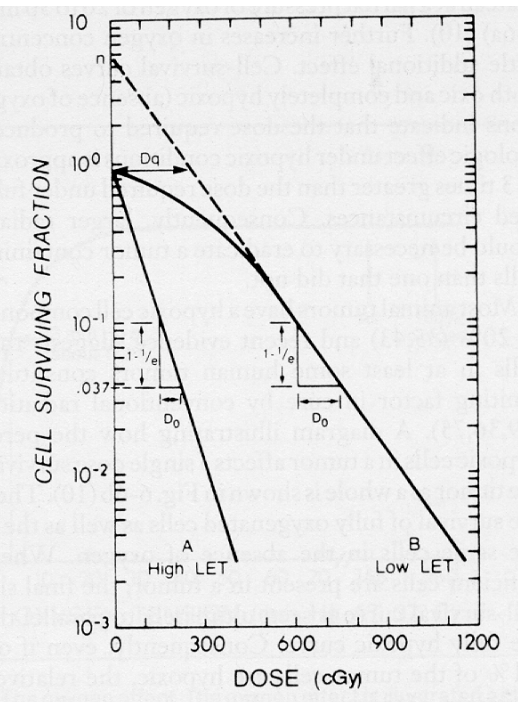

Figure 1-5. A clonogenic cell survival curve represents the fraction or proportion of cells surviving irradiation. On a semilog plot, after an initial shoulder, the curve bends downward sharply and radiation killing become more effective for each cGy absorbed. Cell survival are described by their shoulder $\left(D_{q}\right)$ and slope $\left(D_{0}\right)$ parameters. From Hall [7].

logarithmic or exponential. These curves can be described by their shoulder $\left(D_{q}\right)$ and their slope $\left(D_{o}\right)$. The radiosensitivity is referred to by the $D_{o}$ where $D_{o}$ is required to reduce the survival to $37 \%$ along with the exponential portion of the curves. With this understanding, we can now look into a very important concept of radiation therapy: dose-time relationship.

The magnitude of the radiation dose is not the only-important variable. Of equal importance is the distribution of the dose with respect to time- the fractionation factor. Figure 1-6 shows an idealized fractionation experiment by Elkind and Whitmore using cultured cells in vitro [8]. The survival curve for single acute exposure of x-rays is

Curve A. Curve $\mathrm{S}$ is obtained if the total dose is given as a series of small fractions of the size D1, with the time interval between fractions sufficient for repair of sublethal damage to take place. From this, it is evident that a much larger total dose of radiation is required to achieve the same degree of killing when it is given in a series of small fractions as compared 


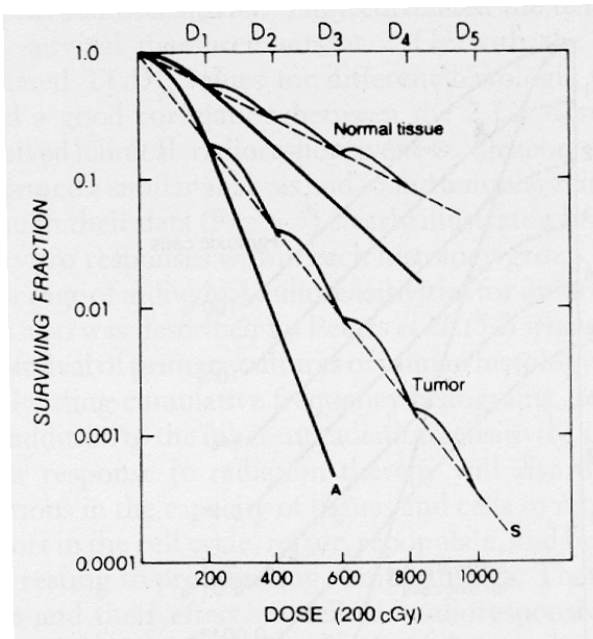

Figure 1-6. Cell repair and fractionation effect. Each repeated fractional dose allows for cell repair of sublethal damage and is expressed by recapitulation of the shoulder of the survival curve. If 1,000 cGy is given as a divided fractional dose (D1, D2, D3, D4, D5) of 200 cGy daily, it achieves a similar degree of cell kill (S) as $600 \mathrm{cGy}$ given in one single exposure (A). From Elkind et al.[8].

with a single large exposure. Finally, clinical experience has shown that normal tissues recover more rapidly than neoplastic tissues. Therefore, by increasing the total dose over a longer period of time, the differential between the tumor lethal dose and tissue tolerance dose can be lessened.

The prescribed dose for different human tumors is not a fixed dose, but varies with tumor size and extent, tumor type, pathologic grade and differentiation, and its response to irradiation. The tumor lethal dose may be defined as the dose that has a $95 \%$ probability of achieving tumor control (cure) - TCD95. Highly radiocurable tumors such as seminomas, lymphosarcomas, Hodgkin's disease, and medulloblastomas are treated in a dose range 3.5 to 6 Gy (TCD95) [6]. Radiocurable tumors (TCD95 range 6 to $7.5 \mathrm{~Gy}$ ) are those requiring the risk of exceeding normal tissue tolerance to assure a high degree of curability [6]. These are moderate to large tumors of the oral cavity, pharynx, bladder, cervix, uterus, ovary, and lung. The least radiocurable tumors (TCD95 range 8 Gy and above) are those large to massive squamous cell and adenocarcinomas that are less consistently cured by irradiation alone and in which the $95 \%$ level is not reached [6]. Some of the recent breast cancer data confirm that tumor control of massive tumors is possible with doses higher than $7 \mathrm{~Gy}$, but such radiation doses are associated with a high incidence of late normal tissue damage. Therefore, radiation oncologists and physicists must decide on an 
appropiate radiation dose that will minimize normal tissue damage but be effective enough to eradicate the tumor.

\section{Effects of Ionizing Radiation}

Normal tissue responds to irradiation at two different levels: 1) acute effects that appear within a short time and 2) late effects that appear months or even years after irradiation. The higher the fractional and total dose and the shorter the time of exposure, the more rapid the onset of tissue necrosis with many cellular compartments destroyed [19]. In this regard, there is always a dose-limiting tissue in the radiation field, and in most cases, it is the vasculoconnective tissue component [19]. Although direct parenchymal cellular depletion of an organ occurs after irradiation, it is often the microvascular circulation that rapidly occludes and infarcts tissues leading to necrosis [19]. This slows the transport of oxygen and vital metabolites and, in turn, contributes to the parenchymal cell depletion, hypoplasia and an eventual late effect [19]. Studies have shown that endothelial cells and smooth muscle cells are gradually lost from the wall of blood vessels after irradiation [20]. These findings also suggest that the amount of depletion is dose-related and endothelial cell loss occurs earlier than that of smooth muscle cells.

In slowly repopulating or non-repopulating tissues, the production of parenchymal hypoplasia is due both to direct cell effects and indirect effect of the microvascular tissue stroma. In both circumstances, the slowly progressive arteriolar fibrosis and interstitial fibrosis after irradiation contribute to the delayed parenchymal hypoplasia and cause the late effects of radiation [19]. Furthermore, lesions occur in the endothelium of small vessels such as arterioles, capillaries, and venules. The resulting lesions are a consequence of inherent radiosensitivity of the relevant cells, smaller diameter of the vessels for a given tissue, or direct organ damage from 
parenchymal cell injury [19]. The swelling of endothelial cells leads to a narrowing obstruction which impedes blood flow. This leads to thrombosis and regenerative attempts by the endothelial cells. In another study, radiation instantaneously increased the vascular permeability of endothelial cells even at doses of 2 Gy [21]. However, when examined at 16 to 24 hours post irradiation, increased permeability was found at and beyond 8 Gy [21]. At 30 days post irradiation, injury was not apparent up to $15 \mathrm{~Gy}$. Thus there appeared to be an instantaneous injury at the capillary level due to irradiation, which appeared to be dose related. Finally, numerous investigations have substantiated one consistent change after irradiation of tissues: the appearance of irregularly spaced vascular constrictions, particularly in the walls of arterioles [19]. The possible development of such constrictions, because of slow depopulation of damaged endothelial cells and reactive hyperplasia to regenerate the loss, can lead to clones of cells at irregularly spaced intervals along the vessel wall, producing a "sausage segment" effect [22].

Radiation has increases leukocyte rolling and adhesion in normal tissue [23]. This suggests that radiation might induce the release of inflammatory mediators to promote leukocyte rolling and adhesion. However, in adenocarcinoma-bearing tissue, radiation did not cause the same changes suggesting that radiation caused changes in the vascular surface properties such that they become less adhesive to leukocytes [23].

\section{Microvascular Networks}

Individual microvascular networks are the basic functional building blocks of the human vascular system [24]. Microvascular networks are defined as a series of interconnected arterioles, capillaries, and venules with low Reynolds number flow and large surface area providing a good environment for the supply of oxygen, nutrients, and the removal of waste 
products. Microvascular networks are the most sensitive part of the vasculature to ionizing radiation. The interaction of blood cells (i.e., RBC, WBC, platelets, etc.) within endothelial cells is significant to the function of normal tissue as well as the treatment of disease. Therefore, a good indication of the health of tissue is the health of the surrounding microvasculature. Understanding the mechanisms and the degree by which ionizing radiation damages the microvasculature has important clinical ramifications.

Arterioles can range from 10 to $125 \mu \mathrm{m}$ in diameter and have a multiple-layered membrane. On the other hand, capillaries have diameters ranging from 7 to $20 \mu \mathrm{m}$ and consist of a single layer of endothelial cells. Venules are small veins with diameters ranging from 20 to $100 \mu \mathrm{m}$ in diameter, with a wall of smooth muscle cells [1]. Arterioles have been shown to regulate the microvascular network blood flow. Blood is supplied by arterioles and traverses through multiple pathways of capillaries before draining into venules. The velocity of blood within the microvasculature can vary between 100 to $2000 \mu \mathrm{m} / \mathrm{s}$ depending on the type of vessel $[25]$.

Microvascular blood flow is unique because of two phenomena called the Fahraeus and Fahraeus-Lindqvist effects. The Fahraeus effect occurs in small vessels $(\mathrm{d}<300 \mu \mathrm{m})$ due to a decrease in the velocity of blood plasma relative to the red blood cell's velocity $[1 ; 26]$. For this reason, the hematocrit inside the vessel is less than the systemic hematocrit. The FahraeusLindqvist effect states that a decrease in the effective viscosity of blood in small vessels due to a decrease in vessel hematocrit results from a decrease in vessel diameter [27]. Therefore, when analyzing hemodynamic parameters one must consider these phenomena to have reliable results.

The interconnectedness of the microvasculature is vital to its function, allowing blood cells to migrate throughout the tissue. Studying complete microvascular networks provides 
information that can not be obtained from analyzing randomly selected vessels. The high degree of interconnectivity and intravascular communication, coordination, and feedback exhibited in microvascular networks requires an integrated approach to microvascular studies. In this project the late effects of ionizing radiation on the structure and function of microvasculature networks in the cremaster muscle of hamsters will be examined. By comparing structural and functional data from the microvascular networks in irradiated and control tissue, we are able to assess the microvascular damage caused by ionizing radiation. 


\section{Chapter 2. OBJECTIVE}

In the clinical arena, radiation oncologists and physicists try to find the best therapeutic dosage of radiation for cancer patients. Too high of a dose will damage the normal tissue while too low of a dose will not eradicate the tumor. In any case, direct exposure to ionizing radiation leads to normal tissue damage. The extent of this normal tissue damage is the focus of our study.

\section{Specific Aims}

The main objective of this research was to quantify the late effects of ionizing radiation $(60,120$, and 180 days post-irradiation) on the structure and function of microvascular networks in normal tissue. This study complements a previous study on the early effects of irradiation (3, 7, and 30 days post-irradiation) which was performed to obtain greater insight into the radiation damage incurred by normal tissue [1-3]. A second objective was to determine whether the methods, protocols, and procedures used in our early effects study would also be useful in this late effects study.

\section{Hypothesis}

Therapeutic doses of ionizing radiation significantly alter the structure and function of microvascular networks in normal tissue at late time points. This hypothesis assumes that microvascular networks are the functional unit of the vascular system and that normal tissue damage is a major dose limiting factor.

Secondly, the protocols used in the early effects study will provide an effective method to examine the late effects of ionizing radiation on normal tissue. 


\section{Key Questions}

1. To what extent are structural (e.g., vessel diameter and length) and functional (e.g., RBC velocity and vessel wall shear stress) parameters of microvascular networks influenced by ionizing radiation?

2. Do changes in structural and functional parameters of microvascular networks found in the late effects study differ from the changes observed in the early effects study?

3. Does the extent of change in structural and functional parameters of irradiated networks vary from vessel to vessel (i.e., arterioles, capillaries, venules)? 


\section{Chapter 3. MATERIALS AND METHODS}

Many aspects of the techniques, procedures, and experimental apparatus described in this section have been described in detail elsewhere [1-3]. The methods described herein were used to quantitatively study the late effects of ionizing radiation on the structure and function of normal tissue microvascular networks at 60,120 , and 180 days post-irradiation. These methods were used in a previous study which examined the early effects of ionizing radiation at 3, 7, and 30 days post-irradiation [1-3]. Therefore, only a summary of the materials and methods will be discussed here.

\section{Experimental Design}

A hamster cremaster muscle model was used to examine the effects of ionizing radiation on microvascular networks in normal tissue. The cremaster muscle is thin and transparent with only two layers of vasculature which facilitates the use of intravital microscopy techniques. The hamsters were 52 days of age (adolescent) and weighed approximately 85 grams at the time of irradiation, which allowed for long term observations (i.e., 180 days) post-irradiation. Agematched control animals were used to account for changes in the microvasculature due to aging.

The hamster cremaster muscle was locally irradiated with a single 10 Gy dose. The cremaster muscle was observed 60,120 , and 180 days post-irradiation. These time points are representative of the late responses of microvascular networks in normal tissue due to radiation exposure. Microvascular networks composed of precapillary arterioles, capillary, and postcapillary venules were observed. Networks were chosen in reference to a well-defined location in the tissue to reduce heterogeneity due to spatial variations. Vessels within the network were given a topological classification of type 1 (precapillary arteriole), type 2 (capillary), and type 3 
vessels (post capillary venule) based on the their two bifurcation ends. Type 1 vessels have both diverging bifurcation ends while type 2 vessels have one diverging and one converging bifurcation. Type 3 vessels consist of two converging bifurcations. The experimental design is shown graphically in Figure 3-1.

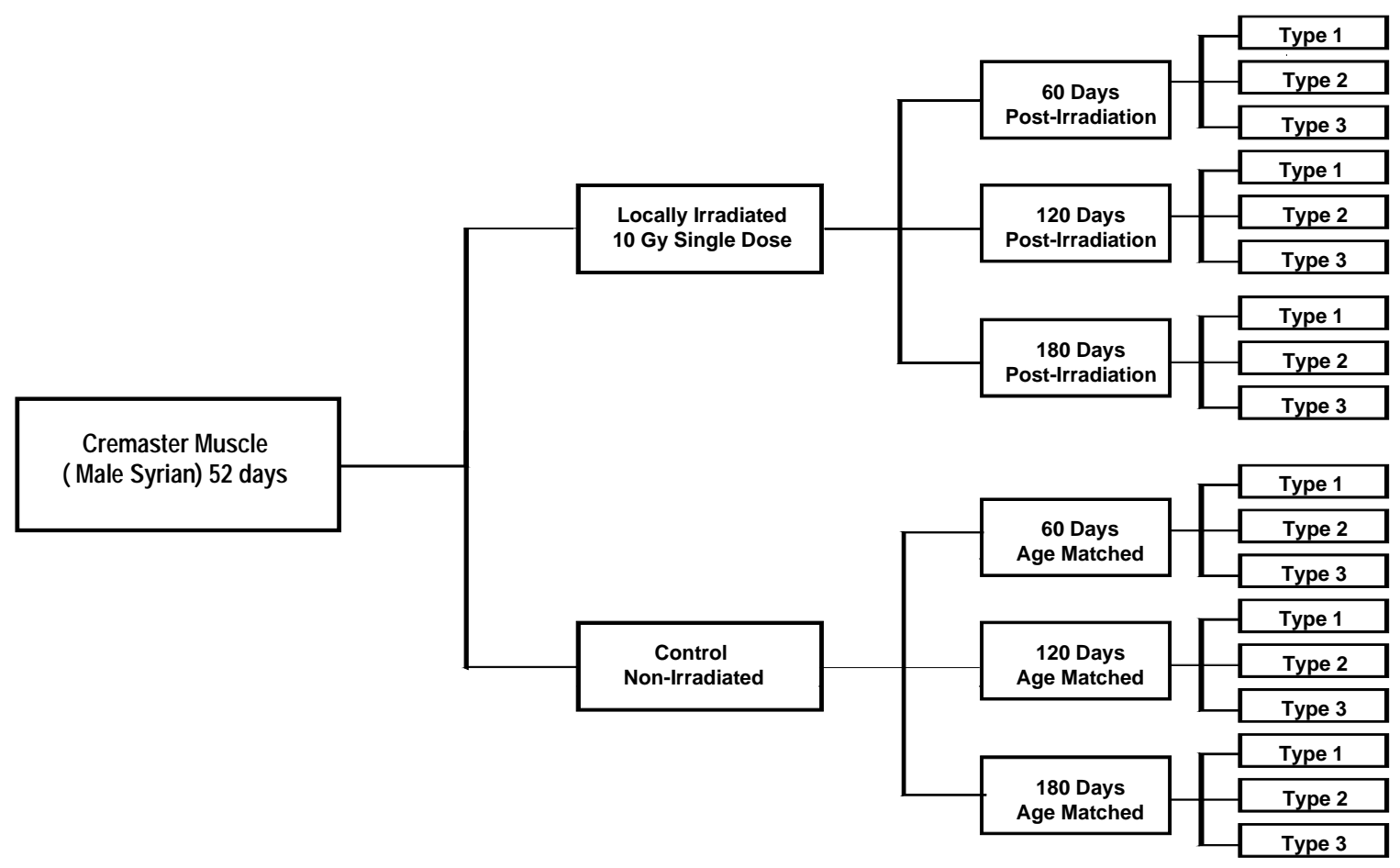

Figure 3-1. Experimental design used to study the late effects of ionizing radiation exposure of normal tissue microvascular. Adapted from Roth [1].

\section{Irradiation}

While the animal was anesthetized ( $25 \mathrm{mg} / \mathrm{kg}$ of Pentobarbital Sodium solution), a single local 10 Gy dose was delivered to the testicles at a rate of $2 \mathrm{~Gy} / \mathrm{min}$ using a Siemens MD-2 linear accelerator (6 mV X-rays). Dose calculations and calibrations were performed by Dr. Sujit Ray (Radiation Physicist at the Regional Medical Center, Memphis, TN) and Dr. Marc Sontag (Radiation Physicist at St. Jude Children's Research Hospital, Memphis, TN). After irradiation, animals were returned back to their cages. 


\section{Surgical Methods}

The surgical methods described were a modification of a cremaster muscle preparation as described by Baez (4). Prior to surgery, animals were anesthetized with an intraperitoneal injection consisting of a mixture of $0.6 \mathrm{~g} / \mathrm{kg}$ urethane and $0.09 \mathrm{~g} / \mathrm{kg} \alpha$-chloralose. During surgery, each animal's body temperature was maintained at approximately $37^{\circ} \mathrm{C}$. While the animal was intubated, an intravenous catheter was inserted in the left femoral vein and used to administer anesthesia and fluorescently labeled RBCs. The animal was placed on a surgical board where the right cremaster muscle was pinned flat with minimal disruption. To simulate physiological conditions, the exposed tissues were superfused at a rate of $5 \mathrm{ml} / \mathrm{min}$ with bicarbonate buffered salt solution equilibrated with $5 \% \mathrm{CO}_{2}-95 \% \mathrm{~N}_{2}$ and maintained at $35 \pm 0.5^{\circ} \mathrm{C}$.

\section{RBC Fluorescent Labeling}

Whole blood was taken by cardiac puncture from age-matched donor animals and was washed and centrifuged three times with a hepes buffer solution to separate the RBCs and remove the buffy coat and white blood cells. Packed RBCs were diluted in $10 \mathrm{ml}$ Hepes buffer solution with albumin and mixed with $0.1 \mathrm{ml}$ of DiI fluorescent dye. The solution was gently stirred using a $1 \mathrm{~cm}$ micro magnetic stirrer for one hour and then washed and centrifuged three

more times to remove excess fluorescent dye. Fluorescently labeled RBCs were delivered to the experimental animals via a left femoral vein catheter. 


\section{Identification of Microvascular Networks}

Once the muscle was pinned, the tissue preparation was allowed to stabilized for 30 minutes before taking data. This allows sufficient time for the tissue to recovery from any stresses incurred during surgery. During this time $0.1 \mathrm{ml}$ of fluorescently labeled RBCs were slowly injected using a standard $1 \mathrm{cc}$ syringe via the femoral vein catheter. In the mean time, a syringe pump was used to infuse a constant supply of anesthesia (36 \% anesthesia and 64\% isotonic saline, delivered at $0.56 \mathrm{ml} / \mathrm{hr}$ ) throughout the remainder of the experiment.

A few drops of a $10^{-4} \mathrm{M}$ adenosine solution were applied topically to the preparation to test for the presence of vascular tone in the second order arteriole supplying the network. A vasodilation of at least $15 \%$ was required for the continuation of the experiment. Complete microvascular networks were chosen in reference to a well-defined location (distal end of second major arcade arteriole to bifurcate from the central feed arteriole) in the tissue to reduce heterogeneity due to spatial variation [28].

\section{Intravital Microscopy and S-VHS Recordings}

Intravital microscopy techniques were used to observe microvascular networks. An industrial grade microscope consisting of trans-illumination (Opti-Quip 75 Watt xenon light source Model No. 1500, Highland Hills, NY) and epi-illumination (Nikon 150 Watt mercury lamp Model No. HB-10101AAF, Japan) light sources was used. The microscope contained a variety of neutral density filters which were used to adjust the light intensity at different magnifications. In addition, a heat filter was incorporated in the light path reduce trauma to the tissue. Two objective lenses (dry 10X and 20X water immersion) were used in conjunction with the $10 \mathrm{X}$ eye pieces. 


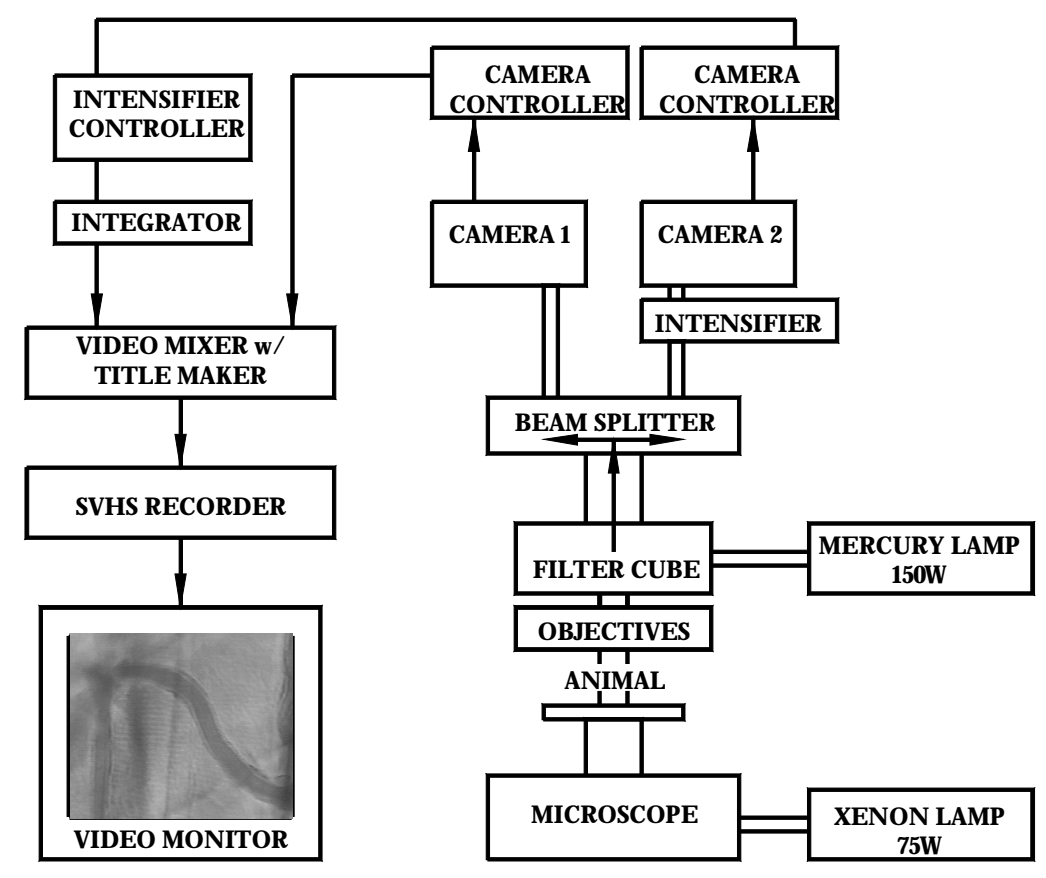

Figure 3-2. Block diagram of video microscopy system. Arrows indicate the signal pathway. Camera 1 and 2 represent the Hamamatsu CCD and MTI CCD72 cameras, respectively. From Roth [1].

The primary camera assembly consisted of a CCD camera (MTI CCD Model No. CCD72, Michigan City, IN) with an intensifier (MTI GenIISys Model No. 104722-01, Michigan City, IN) (see figure 3-2). RBC velocity was measured using the $20 \mathrm{X}$ water immersion lens. A frame integrator was also incorporated into the camera assembly which illuminated all the vessel paths in the frame of view. On average 40 frames were required to get a sharp image of the illuminated vessels. The images were later used to create a collage of images which represented the entire microvascular network.

The secondary camera assembly consisted of a chilled CCD camera and controller (Hamamatsu Model No. C5985, Japan). This camera was used to obtain trans-illumination images with a 20X immersion lens from which vessel diameter was measured. 
The muscle preparation was viewed live on the video monitor and recorded on S-VHS videotapes for off-line analysis. A digital video mixer was used to switch between the camera assemblies. A video title maker was used to place text stamp (date, experimental number, radiation status, and objective lens) on each video field. A time counter was also used to place an electronic time on the video field (see figure 3-2).

\section{Microvascular Maps}

Methods for mapping microvascular networks have been published [2]. The collage of videotaped fluorescent images were digitized using a drawing board (Calcomp DrawBoard III) and AutoCAD software (AutoCAD 14.0 Map Release 2.0) to form maps of the complete microvascular networks. Using GIS (Geographic Information Systems) technology, AutoCAD Map has the unique feature of being able to establish topological and spatial relationships between all vessels in a digitized network. Using a topology query, AutoCAD Map exported information on vessel identification numbers, upstream and downstream nodes, and vessel lengths to an external text file. Microvascular network parameters (i.e., topology, diameter, RBC velocity, etc.) for all vessels in a network were recorded in an external database.

Blood vessels within a microvascular network were separated into three vessel types: type 1, type 2 , and type 3 vessels. Type 1 vessels were defined as vessels with diverging bifurcations at both their upstream and downstream ends. Type 2 vessels were defined as vessels with one diverging and one converging bifurcation whereas type 3 vessels converged at both upstream and downstream bifurcations. No trifurcations were observed.

In addition, a subroutine was written in AutoLisp programming language and run in AutoCAD MAP to color code the vessels of the microvascular network drawing to a specific 
parameter. This allows better representation of the parameters through the use of color. Furthermore, the subroutine also converts the thickness of the vessels to its corresponding diameter by importing data into AutoCAD MAP from an external database (Microsoft Excel 97). The details on how to run the subroutine and the text of the subroutine can be found in Appendix A.

\section{Data Collection}

At the end of each experiment, whole blood was collected into capillary tubes by toe clip. The capillary tubes were placed in a microcentrifuge to measure systemic hematocrit. In addition, a drop of blood was placed in $15 \mathrm{ml}$ of Hepes buffer with albumin to determine the labeled RBC fraction by flow cytometry (Coulter Epics Profile II, Miami, FL).

Measurements of vessel diameter, RBC flux, and RBC velocity for all vessels within a microvascular network were made using a video caliper system. Vessel diameters were analyzed off-line from S-VHS videotapes using an interactive routine developed within the MetaMorph environment. Vessel diameter was considered to be the distance between the outside borders of endothelial cells comprising the vessel wall. To reduce the variability in the diameter of the vessel, multiple measurements were made at $20 \mu \mathrm{m}$ intervals along the vessel length. A simple average was used to estimate the diameter of a vessel.

Using the MetaMoph system for image analysis, RBC velocity measurements were taken from fluorescent images. First, an image was acquired, and a marker was placed at the tip of a labeled RBC. The videotape was then advanced a set number of frames and a second image with the original marker was acquired. A line is drawn from the original marker to the tip of traveled RBC. The line represented the distance traveled by the $\mathrm{RBC}$ which was measured by 
MetaMorph and automatically logged in a database (Microsoft Excel). To account for the statistical variability in $\mathrm{RBC}$ velocity, fifteen measurements were taken for each vessel, and a simple average was calculated.

RBC flux was measured by counting the number of fluorescently labeled RBCs crossing a section of vessel per unit time. Flux measurement was made possible by using a time stamp placed on the images. The videotape was advanced a set amount of time while the number of labeled RBCs was measured using a hand counter (Compass Counter, Taiwan). The labeled RBC flux was divided by the labeled cell fraction (obtained by flow cytometry) to obtain a true measure of RBC flux.

\section{Calculated Parameters}

Hemodynamic parameters that could not be measured directly were calculated using the data collected above.

The tortuosity of a vessel was estimated as the straight length divided by the actual length (distance between end nodes of a vessel) and was computed as

$$
T=1-\frac{\text { Straight_Length }}{\text { Actual_Length }} \quad \text { Equation 3-1 }
$$

Vessel hematocrit was calculated using the following equation:

$$
H t=\frac{4 f_{R B C} V_{c}}{V_{R B C} \pi d^{2}}
$$

Equation 3-2 
where $\mathrm{V}_{\mathrm{c}}$ is the mean $\mathrm{RBC}$ corpuscular volume, $\mathrm{V}_{R B C}$ is the mean velocity, $f_{R B C}$ is the mean flux, and $d$ is the mean diameter. The mean RBC corpuscular volume for a hamster is assumed to be approximately $70 \mu \mathrm{m}^{3}$. This equation assumes a circular cross-section for all vessels whereas lineal density is independent of that assumption. Lineal density is the number of cells per unit length at an instant in time and estimated using the following equation:

$$
L_{D}=\frac{f_{R B C}}{V_{R B C}}
$$

Equation 3-3

Measurements of vessel hematocrit and lineal density varied differently and thus both were reported.

Due to the size of the microvessels, a parametric equation based on the Fahraeus effect was used to estimate discharge hematocrit. This equation which was used for rat blood describes the change in discharge hematocrit $\left(\mathrm{H}_{\mathrm{D}}\right)$ relative to tube hematocrit $\left(\mathrm{H}_{\mathrm{t}}\right)[29]$.

$$
\frac{H_{t}}{H_{D}}=H_{D}+\left(1-H_{D}\right) \bullet\left(1+1.7 e^{-0.415 d}-0.6 e^{-0.011 d}\right) \quad \text { Equation 3-4 }
$$

After scaling the diameter exponents with the cube root of mean RBC corpuscular volume for hamster blood (Rat: $61 \mu \mathrm{m}^{3}$, Hamster: $70 \mu^{3}$ ) and solving for discharge hematocrit we obtained

$$
H_{D}=\frac{-R \pm \sqrt{R^{2}+4 H_{t}(1-R)}}{2(1-R)}
$$

Equation 3-5

where,

$$
R=1+1.7 e^{-0.4762 d}-0.6 e^{-0.0126 d}
$$

Equation 3-6 
The equation for discharge hematocrit is sensitive to the assumptions of circular cross-section and diameter measurements for each vessel.

The effective blood viscosity in vivo is a parameter that changes with flow conditions (i.e., vessel diameter, hematocrit, etc.). A parametric equation taking into account the variations of relative effective viscosity with discharge hematocrit and vessel diameter for rat blood was developed by Pries et al [30],

$$
\eta_{\text {rel }}=1+\frac{e^{H_{D} \bullet \alpha}-1}{e^{0.45 \bullet \alpha}-1} \bullet\left(110 e^{-1.424 d}+3-3.45 e^{-0.035 d}\right) \quad \text { Equation } 3-7
$$

where

$$
\alpha=\frac{4}{1+e^{-0.593 \bullet(d-6.74)}}
$$

Equation 3-8

Again, scaling the diameter exponents in Equation 3.7 and 3.8 for hamster blood, and multiplying Equation 3.8 by the viscosity of plasma in hamster blood at $37^{\circ} \mathrm{C}$ we obtained,

$$
\eta_{\text {eff }}=1.16\left|1+\frac{e^{H_{D} \bullet \alpha}-1}{e^{0.45 \bullet \alpha}-1} \bullet\left(110 e^{-1.63 d}+3-3.45 e^{-0.04 d}\right)\right| \quad \text { Equation 3-9 }
$$

where

$$
\alpha=\frac{4}{1+e^{-0.593 \bullet(1.15 d-6.74)}}
$$

To calculate vessel shear stress, an estimate of the average blood velocity ( $\left.\mathrm{V}_{\text {Blood }}\right)$ was determined using the following relationship which takes in consideration the Fahraeus effect:

$$
V_{\text {Blood }}=\frac{V_{R B C} H_{t}}{H_{D}}
$$


Vessel wall shear stress $\left(\tau_{\mathrm{w}}\right)$ was calculated from the following equation,

$$
\tau_{W}=\eta_{e} \gamma
$$

where the vessel wall shear rate $\gamma$ is given by

$$
\gamma=\frac{8 V_{\text {Blood }}}{d}
$$

Equation 3-13

Combining Equations 3.12 and 3.13, we obtain a measure of vessel wall shear stress,

$$
\tau_{W}=\frac{V_{B l o o d} \eta_{e}}{125 d}
$$

Equation 3-14

Vessel wall shear stress is most sensitive to the accuracy and precision of diameter measurements and is dependent on previously calculated values (Equations 3.7 and 3.11). Average RBC transit time is an important indicator of the oxygen delivery capacity of a microvascular network. The $\mathrm{RBC}$ transit time program consists of four main procedures [1]. First, using network topology all possible paths within a network were determined. Next, entrance probability and travel time were calculated. Entrance probability (EP) is the probability of a RBC entering another vessel at a bifurcation from an upstream parent vessel and was calculated as

$$
E P=\frac{F_{d}}{F_{p}}
$$

Equation 3.15

where $F_{d}$ is the flux of the daughter vessel and $F_{p}$ is the flux at the parent vessel. To satisfy conservation of mass of RBCs, the probability of a vessel which converged at its upstream end was set equal to one. RBC travel time for a vessel was the length of the vessel divided by its $\mathrm{RBC}$ velocity and was given by 


$$
T R=\frac{L}{V_{R B C}}
$$

Equation 3.16

Thirdly, the probability of taking a path was the product of the entrance probabilities (Equation 3.15),for all vessels within that path. On the other hand, the RBC travel time for a path was the summation of the RBC travel time (Equation 3.16) for all vessels with that path. Lastly, the average $\mathrm{RBC}$ transit time was estimated as the summation of the product of the paths probability and $\mathrm{RBC}$ travel time for all paths within that network. Because microvascular networks differ in both the number of vessels and their vascular density, RBC transit time was divided by vascular density to normalize the data.

\section{Statistical Analysis}

A factorial design was used to examine the effects of three factors and their interactions on microvascular network structure and function. The factors and their subdivisions were radiation status (i.e., control and irradiated), time post-irradiation (i.e., 60, 120, and 180 days post-irradiation), and network vessel type (i.e., type 1, type 2, and type 3). Using Statgraphics Plus software (Manugistics, Inc., Rockville, MD), multi-factor analysis of variance (ANOVA) was used to test the effects of these factors and interactions. A multiple comparison procedure (Fisher's least significant difference) was used to discriminate among the means. Differences between the means (control vs. irradiated) were considered statistically significant if $\mathrm{P}<0.05$.

The sample size used in this study was $\mathrm{N}=5$ animals per time point per treatment. The sample size was determined in the early effect study by Noah Roth [1] using a graph of power analysis of variance as a function of a non-dimensional parameter. 


\section{Chapter 4. RESULTS}

Changes in the structural and functional parameters of microvascular networks were examined using a hamster cremaster muscle model at 60,120 , and 180 post-irradiation. The study complements earlier work performed in our laboratory which examined networks at 3, 7, and 30 days post-irradiation [1-3]. In both studies, five animals were used for each time point group per treatment group. From each animal, one complete microvascular network was analyzed. On average, each network contained approximately 37 vessels of which $46 \%$ were type 1 vessels, $35 \%$ were type 2 , and $19 \%$ were type 3 vessels. The number of vessels per time point ranged from 334 to 412 , and the total number of vessels studied was 1,111.

Data were collected for the following parameters: weight, arteriolar tone, vessel diameter, length, $\mathrm{RBC}$ velocity, and RBC flux. From the data collected, the following parameters were calculated: tortuosity, transient time, shear stress, lineal density, vessel hematocrit, and the product of vessel hematocrit and $\mathrm{RBC}$ velocity. The data are presented as Mean $\pm \mathrm{SEM}$ (Standard Error of the Mean). Multifactor analysis of variance (ANOVA) was used to examine the effects of three factors (irradiation, age, and vessel type) and their interactions on structural and functional parameters. Differences were considered statistically significant if $\mathrm{P}<0.05$.

\section{Result Summary}

Animals were irradiated at 52 days of age where they each weighed approximately 85 grams. However after 60 and 120 days, the weights of irradiated animals were significantly less than that of controls (see figure 4-1). At 180 days post-irradiation, irradiated and control animals weighed approximately the same. 


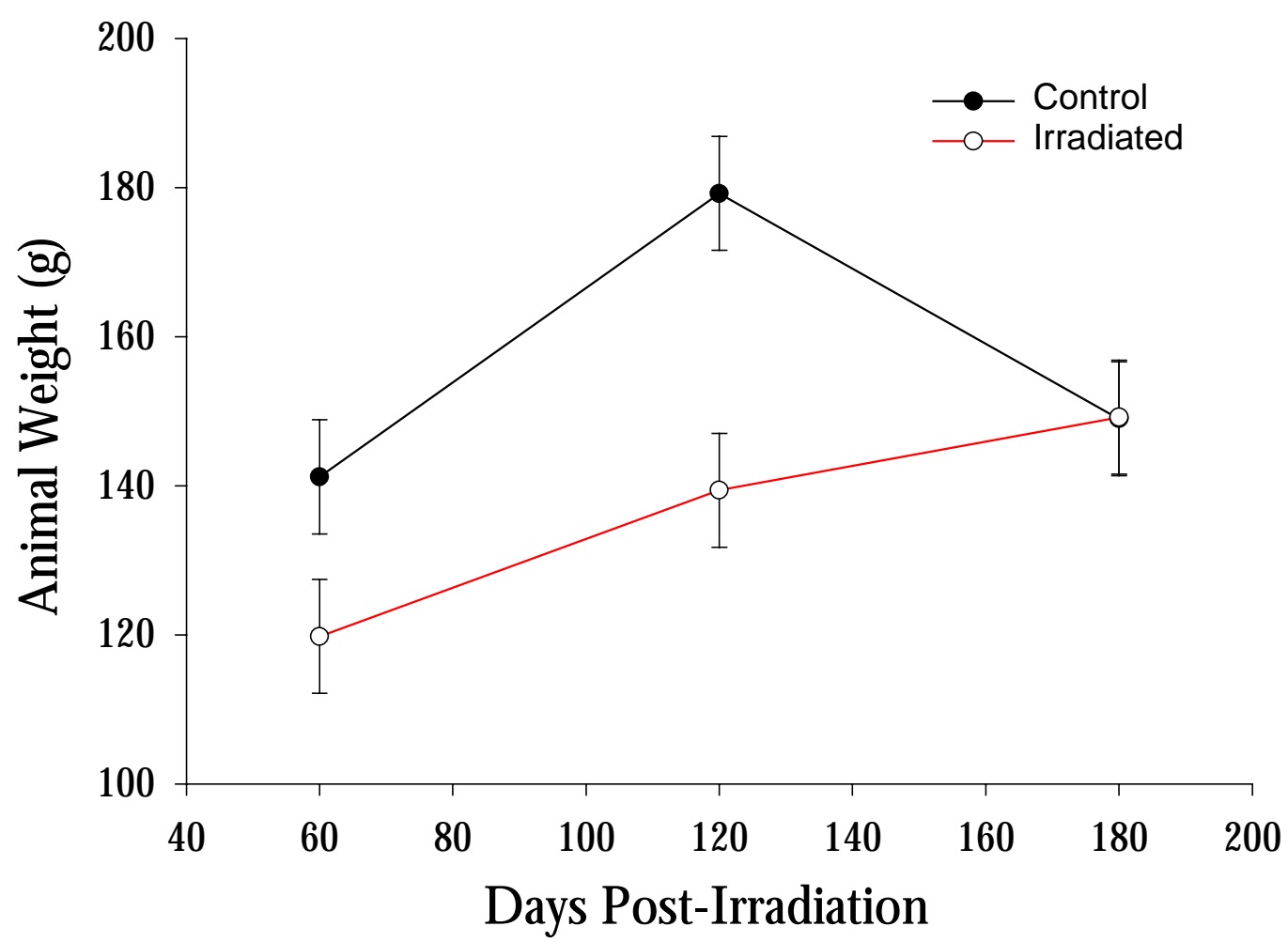

Figure 4-1. Animal weight at $60,120,180$ days post-irradiation $(\mathrm{N}=5$ animals per time point per treatment group). Data are presented as Mean \pm SEM $(\mathrm{P}<0.05)$.

Vascular tone was measured using an application of adenosine solution $\left(10^{-4} \mathrm{M}\right)$ to a second order arteriole supplying each microvascular network, but results show no significant difference between irradiated and controls. In addition, no significant differences in RBC transit time between irradiated and controls were observed. Similarly, the capillary surface area of irradiated vessels was not significantly different from control vessels.

\section{Structural Parameters}

Before discussing the structural parameters, it is important to note that no morphological changes or unusual microvascular formations (e.g., aneurysms) were observed in irradiated animals. The diameter of irradiated vessels significantly greater than controls at 120 days post- 
irradiation. Data also indicate that the diameter of type 3 irradiated vessels were significantly greater than controls for all time post-irradiation (see figure 4-2).

Length of irradiated vessels at 180 days post-irradiation was the only group significantly less than controls. Measurements of tortuosity and vascular density showed no difference between irradiated and control groups.

\section{Functional Parameters}

While average $\mathrm{RBC}$ velocity decreased with age in control animals, the average $\mathrm{RBC}$ velocity in irradiated vessels showed a significant decrease from controls at 120 days postirradiation and then an increase at 180 days post-irradiation (see figure 4-3).

The RBC flux in vessels in irradiated networks was significantly greater than control at 180 days post-irradiation. At 180 days post-irradiation, irradiated animals had higher RBC flux in all vessel types. Although there were no significant differences in RBC flux at 60 and 120 days post-irradiation, the RBC flux in type 3 irradiated vessels was significantly greater than controls at all time points (see tables 4-1,2,3).

The calculated hematocrit of vessels in irradiated networks was significantly different

from controls at 120 and 180 days post-irradiation (see figure 4-4). At 180 days post-irradiation, 


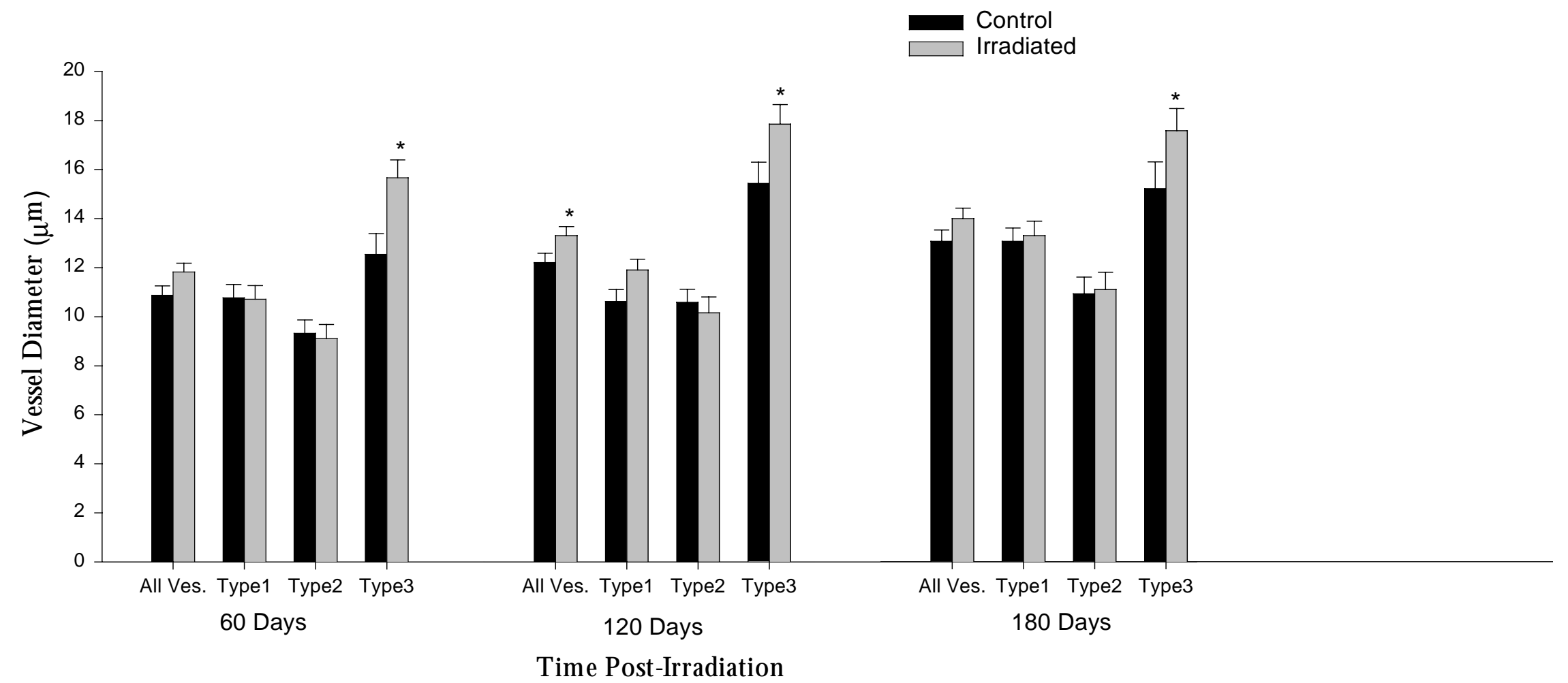

Figure 4-2. Vessel diameter ( $\mathrm{N}=5$ animals per treatment group per time point) of type 1,2,3, and all vessels at 60,120, and 180 days post-irradiation. Data are presented Mean \pm SEM. Significant differences are indicated by $*(\mathrm{P}<0.05)$. 


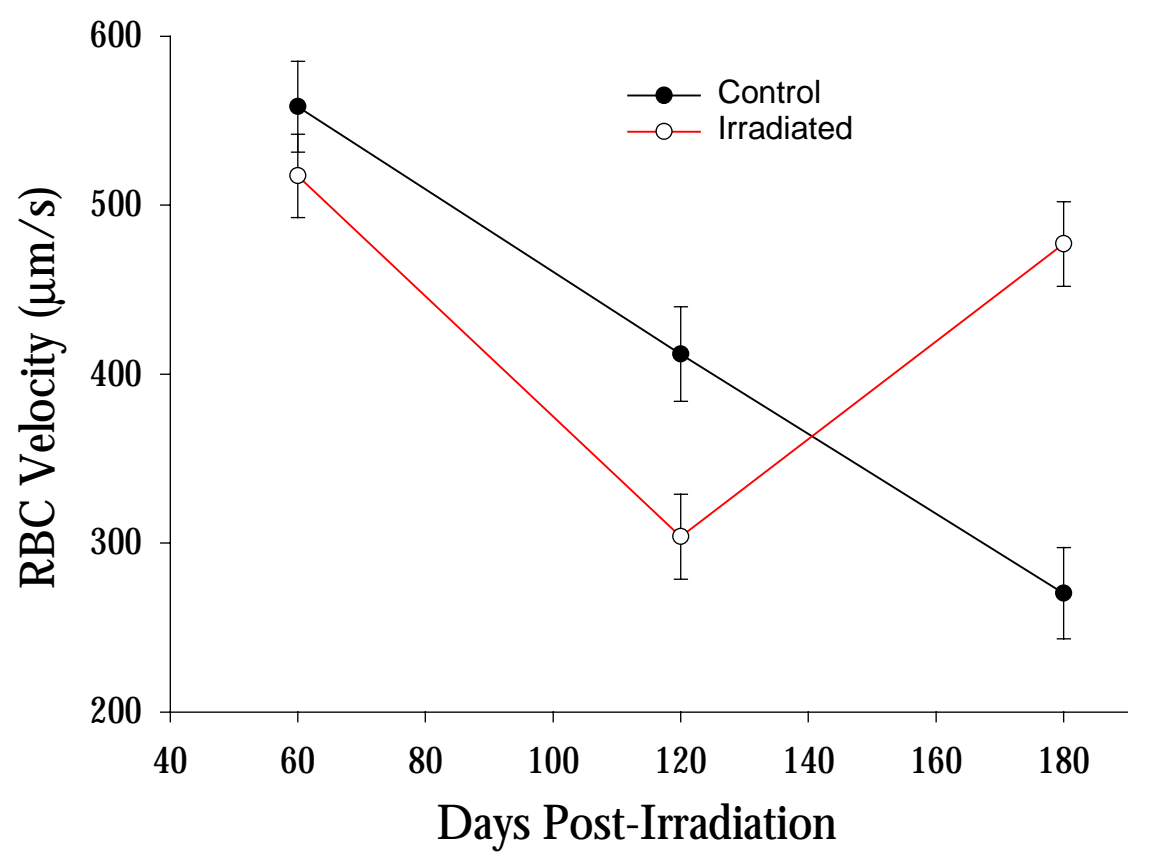

Figure 4-3. RBC velocity at 60, 120, 180 days post-irradiation $(\mathrm{N}=5$ animals per time point per treatment group). Data are presented Mean \pm SEM $(\mathrm{P}<0.05)$.

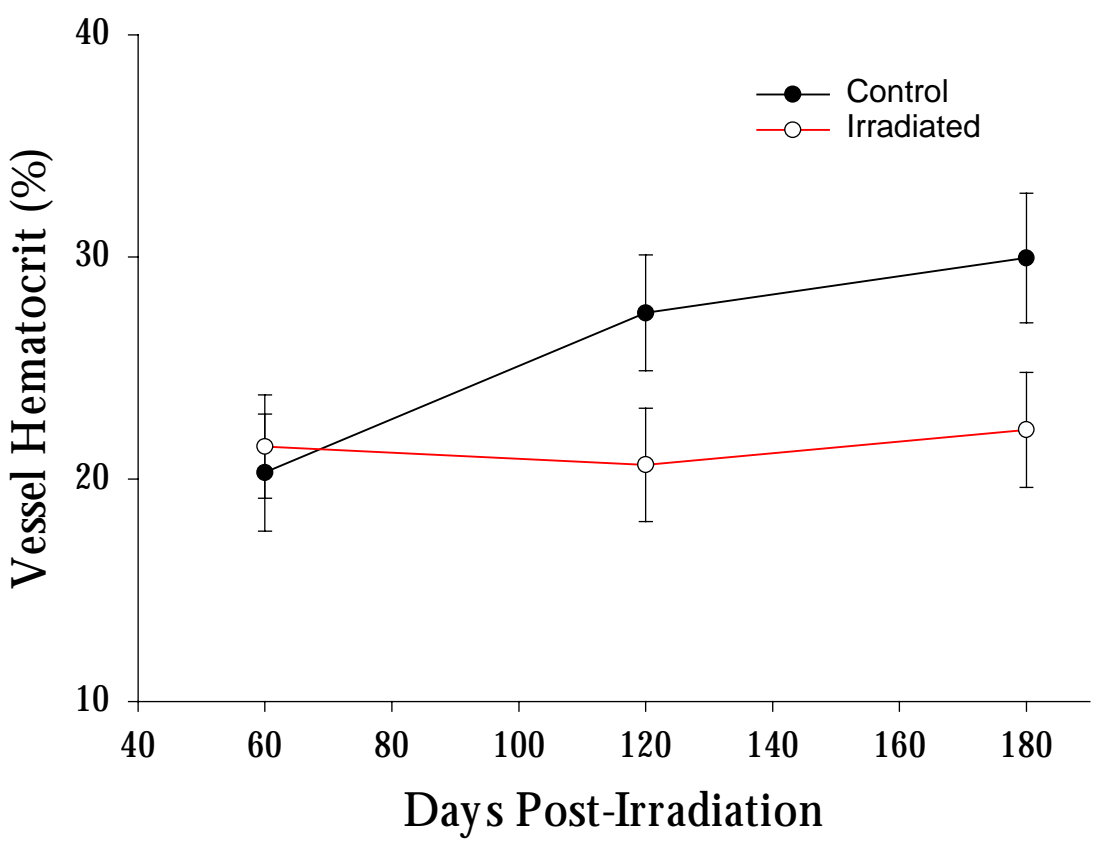

Figure 4-4. Vessel hematocrit at $60,120,180$ days post-irradiation $(\mathrm{N}=5$ animals per time point per treatment group). Data are presented Mean \pm SEM $(\mathrm{P}<0.05)$. 


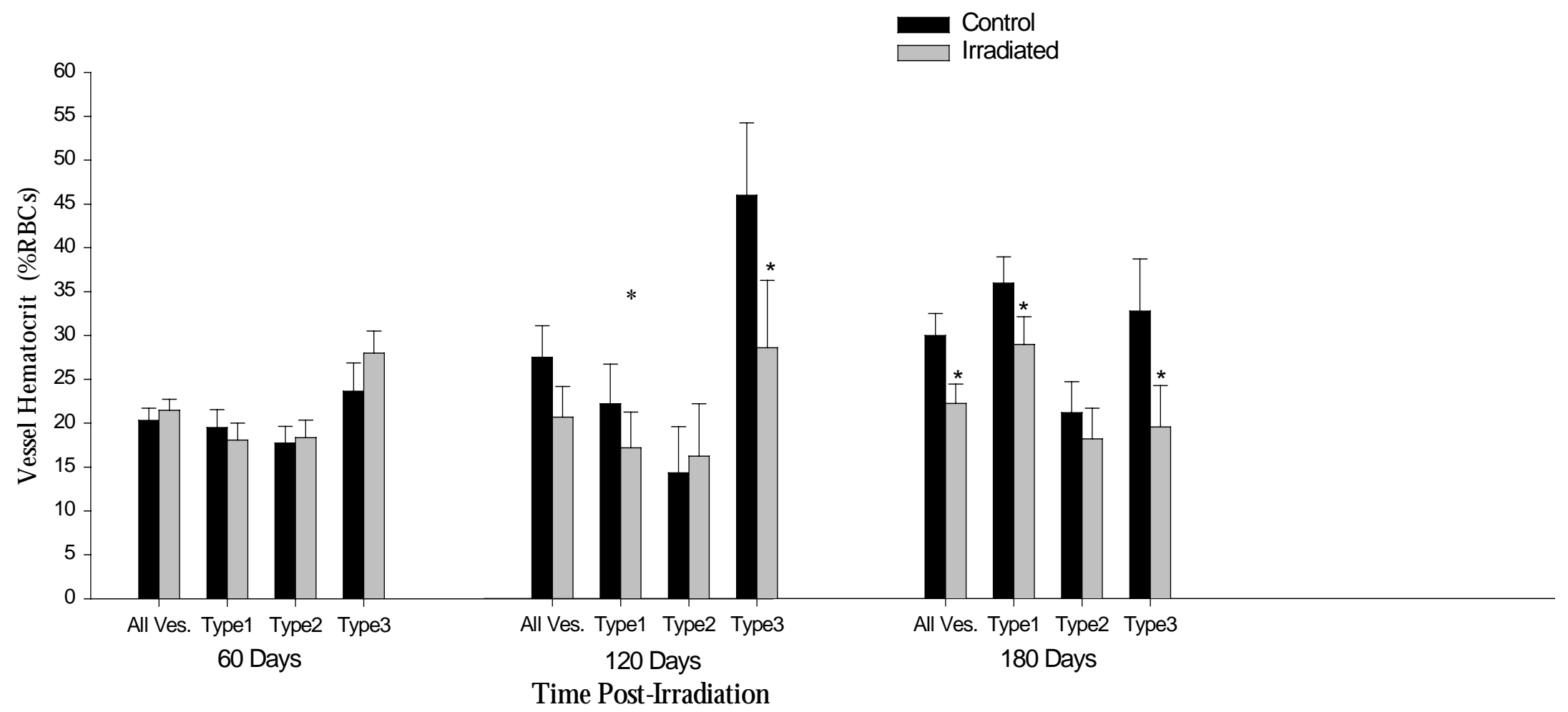

Figure 4-5. Vessel hematocrit $(\mathrm{N}=5$ animals per treatment group per time point) of type $1,2,3$, and all vessels at 60,120, and 180 days post-irradiation. Data are presented Mean \pm SEM. Significant differences are indicated by $*(\mathrm{P}<0.05)$. 


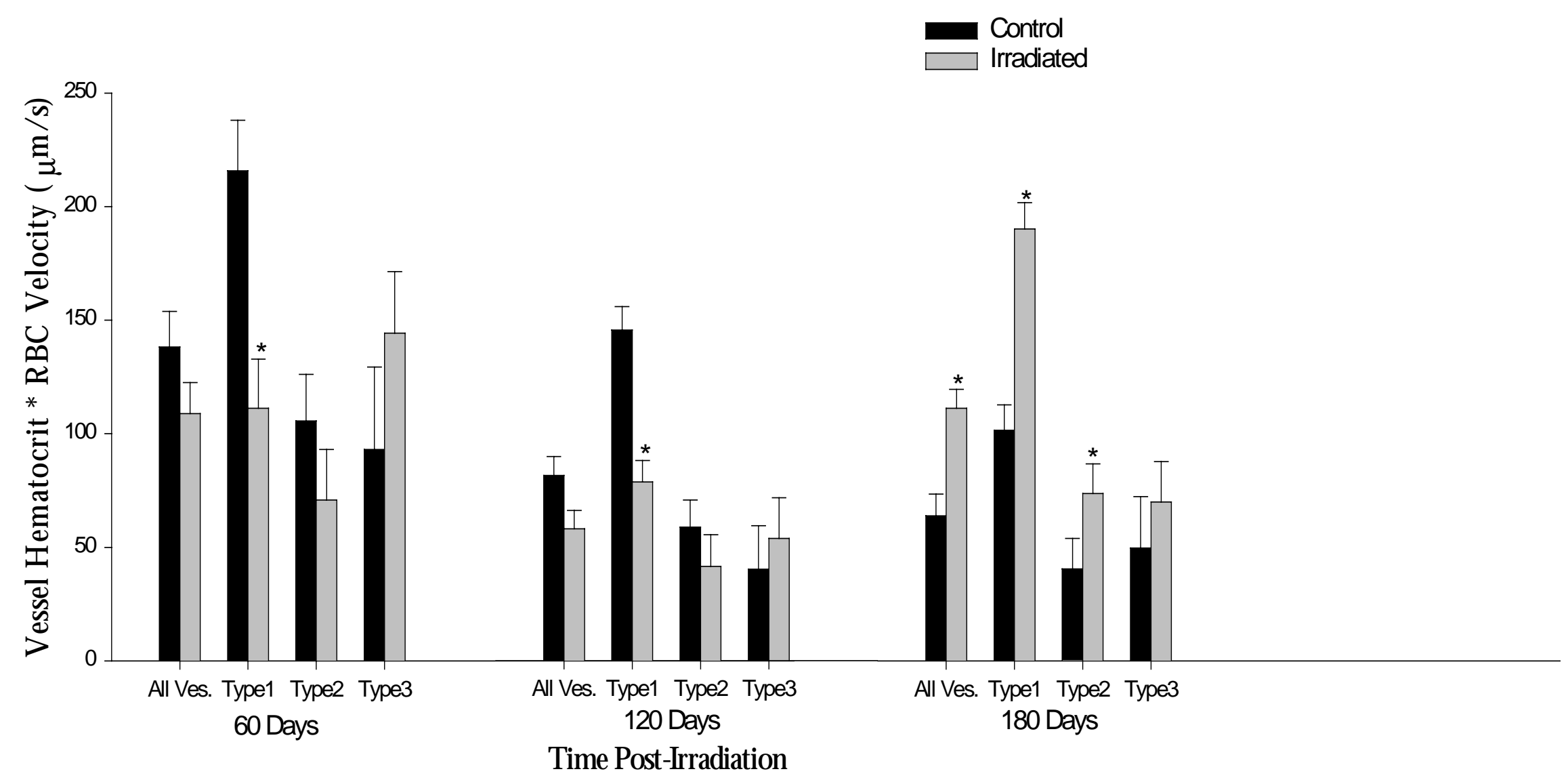

Figure 4-6. The product of vessel hematocrit and $\mathrm{RBC}$ velocity $(\mathrm{N}=5$ animals per treatment group per time point) of type $1,2,3$, and all vessels at 60,120 , and 180 days post-irradiation. Data are presented Mean \pm SEM. Significant differences are indicated by $*(\mathrm{P}<0.05)$. 


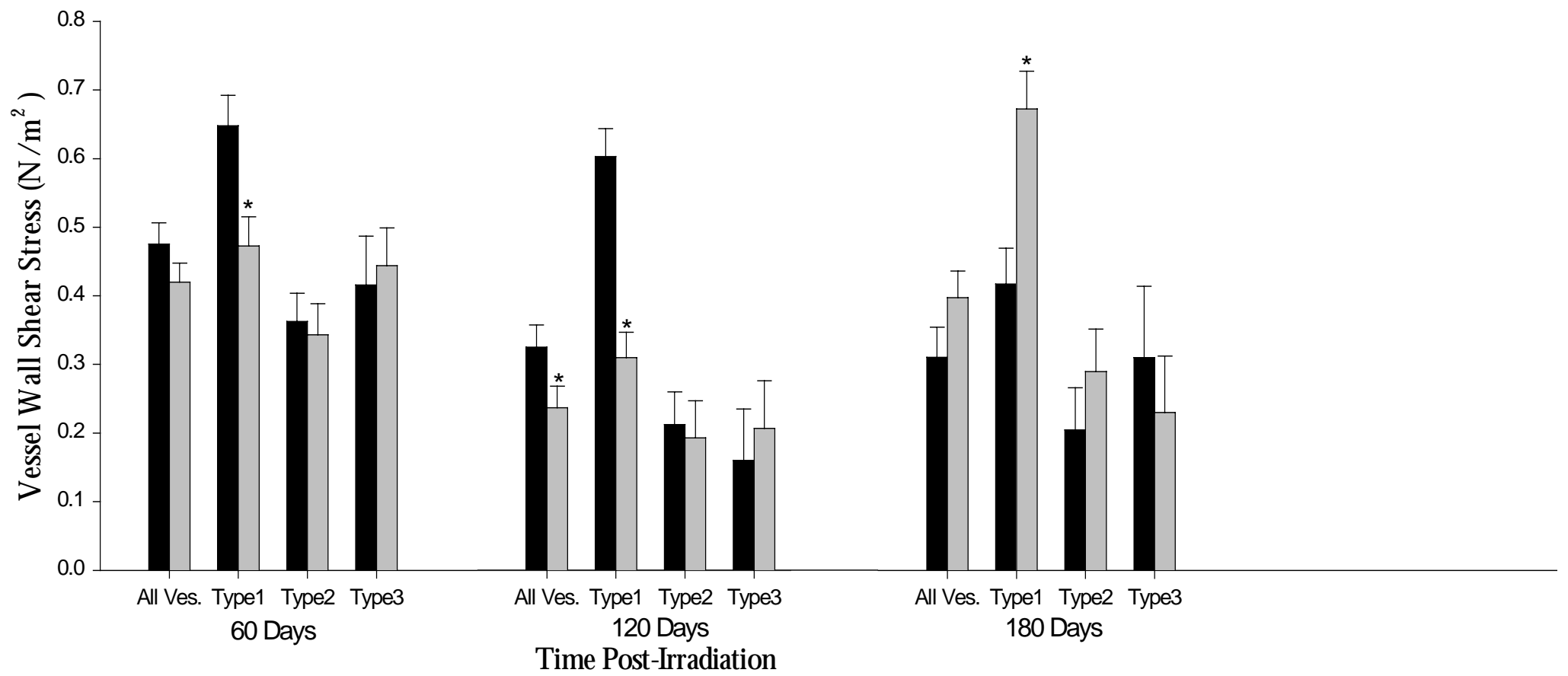

Figure 4-7. Vessel wall shear stress ( $N=5$ animals per treatment group per time point) of type 1, 2, 3, and all vessels at 60,120 , and 180 days post-irradiation. Data are presented Mean \pm SEM. Significant differences are indicated by $*(\mathrm{P}<0.05)$. 
vessel hematocrit was significantly smaller in type 1 and 3 irradiated vessel. At 120 days postirradiation, vessel hematocrit in type 3 irradiated vessel was significantly smaller than control (see figure 4-5). Lineal density, which is the number of RBCs per unit length, was not significantly different between control and irradiated vessels.

The product of RBC velocity and vessel hematocrit, which is an index of oxygen delivery capacity, was significantly different in irradiated and control animals. At 60 and 120 days postirradiation, type 1 irradiated vessels were significantly smaller compared to controls. Meanwhile, at 180 days post-irradiation, the product of RBC velocity and vessel hematocrit was significantly larger in irradiated networks (see figure 4-6).

Calculated vessel wall shear stress in irradiated networks was also significantly different from controls. At 60 and 120 days post-irradiation, type 1 irradiated vessels were significantly smaller than controls. However, at 180 days post-irradiation, type 1 irradiated vessel were significantly greater than control (see figure 4-7).

A summary of all the data collected is presented in the following tables. Table 4-1 is a summary of all the data for each age group. Table 4-2, 4-3, and 4-4 are summaries of data for 60 , 120 , and 180 days post-irradiation, respectively, with subdivisions of type $1,2,3$, and all vessels. Table 4-5, 4-6, and 4-7 contain summaries of data for type 1, 2, and 3 vessels, respectively, with subdivision of time post-irradiation. Highlighted regions represent statistically significant data. Data are presented as Mean +-SEM.

In addition, animal weight, RBC velocity, and vessel hematocrit from our early effects study has been incorporated into Figure B-1, B-2, and B-3 (appendix B) in order to get a better representation of the both early and late effects of ionizing radiation. 


\section{Table 4-1}

The Interactions of Time Post-Irradiation and Radiation Status for All Data

\begin{tabular}{|c|c|c|c|c|c|c|c|c|}
\hline & & \multicolumn{3}{|c|}{$\begin{array}{l}\text { 60 DAYSPOST- } \\
\text { IRRADIATION }\end{array}$} & \multicolumn{2}{|c|}{$\begin{array}{l}\text { 120 DAYSPOST- } \\
\text { IRRADIATION }\end{array}$} & \multicolumn{2}{|c|}{$\begin{array}{l}\text { 180 DAYSPOST- } \\
\text { IRRADIATION }\end{array}$} \\
\hline \multirow[b]{2}{*}{ ANIMAL: } & \multirow[b]{2}{*}{$W(g)$} & CONTROL & \multicolumn{2}{|c|}{ IRRADIATED } & CONTROL & IRRADIATED & CONTROL & IRRADIATED \\
\hline & & $141.2 \pm 7.64$ & \multicolumn{2}{|l|}{$119.8 \pm 7.64$} & $179.2 \pm 7.64$ & $139.4 \pm 7.64$ & $149.0 \pm 7.64$ & $149.2 \pm 7.64$ \\
\hline \multirow[t]{3}{*}{ NETWORK: } & $\mathrm{V}_{\text {Tone }}(\%)$ & $32.6 \pm 7.85$ & \multicolumn{2}{|l|}{$29.6 \pm 7.85$} & $31.06 \pm 7.85$ & $40.5 \pm 7.85$ & $19.88 \pm 7.85$ & $15.54 \pm 7.85$ \\
\hline & $\mathrm{SA}\left(\mu \mathrm{m}^{2}\right)$ & $456317 \pm 70156$ & \multicolumn{2}{|c|}{$465588 \pm 70156$} & $624896 \pm 70156$ & $561623 \pm 70156$ & $599709 \pm 70156$ & $527201 \pm 70156$ \\
\hline & $t_{\mathrm{RBC}}(\mathrm{s})$ & $1.253 \pm 0.352$ & \multicolumn{2}{|c|}{$1.568 \pm 0.352$} & $2.068 \pm 0.352$ & $2.044 \pm 0.352$ & $3.791 \pm 0.352$ & $1.723 \pm 0.352$ \\
\hline \multirow[t]{3}{*}{ STRUCTURAL: } & $\mathrm{d}(\mu \mathrm{m})$ & $10.88 \pm 0.38$ & \multicolumn{2}{|c|}{$11.83 \pm 0.36$} & $12.21 \pm 0.38$ & $13.30 \pm 0.37$ & $13.07 \pm 0.44$ & $13.99 \pm 0.41$ \\
\hline & $\mathrm{L}(\mu \mathrm{m})$ & $159.28 \pm 11.68$ & \multicolumn{2}{|c|}{$162.06 \pm 11.04$} & $177.09 \pm 11.61$ & $157.78 \pm 10.74$ & $221.68 \pm 11.98$ & $185.54 \pm 10.67$ \\
\hline & $\mathrm{T}(\%)$ & $11.72 \pm 1.37$ & \multicolumn{2}{|c|}{$11.00 \pm 1.30$} & $10.89 \pm 1.37$ & $11.65 \pm 1.26$ & $16.30 \pm 1.41$ & $14.97 \pm 1.25$ \\
\hline \multirow[t]{6}{*}{ FUNCTIONAL: } & $\mathrm{V}_{\mathrm{RBC}}(\mu \mathrm{m} / \mathrm{s})$ & $558.2 \pm 26.83$ & \multicolumn{2}{|c|}{$517.3 \pm 24.63$} & $411.8 \pm 27.94$ & $303.71 \pm 25.27$ & $270.27 \pm 26.89$ & $476.92 \pm 25.09$ \\
\hline & $\mathrm{f}_{\mathrm{RBC}}(\mathrm{RBC} / \mathrm{s})$ & $163.41 \pm 16.13$ & \multicolumn{2}{|l|}{$177.6 \pm 15.24$} & $112.52 \pm 16.24$ & $120.55 \pm 14.83$ & $127.77 \pm 16.70$ & $197.65 \pm 15.81$ \\
\hline & $\mathrm{H}_{\mathrm{t}}(\%)$ & $20.30 \pm 2.62$ & \multicolumn{2}{|c|}{$21.46 \pm 2.32$} & $27.48 \pm 2.60$ & $20.64 \pm 2.55$ & $29.95 \pm 2.91$ & $22.21 \pm 2.58$ \\
\hline & $\mathrm{L}_{\mathrm{D}}(\mathrm{RBC} / \mu \mathrm{m})$ & $0.328 \pm 0.052$ & \multicolumn{2}{|c|}{$0.378 \pm 0.047$} & $0.502 \pm 0.054$ & $0.490 \pm 0.049$ & $0.508 \pm 0.052$ & $0.429 \pm 0.049$ \\
\hline & $\mathrm{V}_{\mathrm{RBC}} \mathrm{H}_{\mathrm{t}}(\mathrm{RBQ} \mu \mathrm{m} / \mathrm{s})$ & $138.1 \pm 11.48$ & \multicolumn{2}{|c|}{$108.8 \pm 10.01$} & $81.56 \pm 11.44$ & $58.06 \pm 11.28$ & $63.88 \pm 12.86$ & $111.2 \pm 11.30$ \\
\hline & $\tau_{\mathrm{W}}\left(\mathrm{N} / \mathrm{m}^{2}\right)$ & $0.475 \pm 0.034$ & \multicolumn{2}{|l|}{$0.420 \pm 0.030$} & $0.325 \pm 0.034$ & $0.236 \pm 0.033$ & $0.311 \pm 0.038$ & $0.397 \pm 0.034$ \\
\hline \multirow{2}{*}{\multicolumn{4}{|c|}{$\begin{array}{l}\text { Significant Difference between CN and IR } \\
\qquad \mathrm{P}<0.01\end{array}$}} & \multicolumn{5}{|c|}{ Data are presented as Mean \pm SEM, $N=5$ animals, and $n=1110$ vessels } \\
\hline & & & & \multicolumn{5}{|c|}{ No Significant Difference between CN and IR } \\
\hline
\end{tabular}


Table 4-2

The Interactions of Vessel Type and Radiation Status for 60 Days Post-Irradiation

\begin{tabular}{|c|c|c|c|c|c|c|c|c|}
\hline \multirow{3}{*}{$\begin{array}{l}\text { STRUCTURAL: } \\
\text { d }(\mu \mathrm{m})\end{array}$} & \multicolumn{2}{|c|}{ ALL VESSELS } & \multicolumn{2}{|c|}{ TYPE 1} & \multicolumn{2}{|c|}{ TYPE 2} & \multicolumn{2}{|c|}{ TYPE 3} \\
\hline & CONTROL & IRRADIATED & \multirow{2}{*}{$\begin{array}{l}\text { CONTROL } \\
10.77 \pm 0.55\end{array}$} & IRRADIATED & CONTROL & IRRADIATED & CONTROL & IRRADIATED \\
\hline & $10.88 \pm 0.38$ & $11.83 \pm 0.36$ & & $10.72 \pm 0.54$ & $9.327 \pm 0.54$ & $9.102 \pm 0.58$ & $12.54 \pm 0.84$ & $15.66 \pm 0.73$ \\
\hline $\mathrm{L}(\mu \mathrm{m})$ & $159.3 \pm 10.9$ & $162.1 \pm 10.3$ & $126.5 \pm 16.1$ & $116.3 \pm 16.4$ & $226.3 \pm 15.8$ & $262.2 \pm 16.8$ & $125.0 \pm 23.6$ & $107.6 \pm 20.0$ \\
\hline $\mathrm{T}(\%)$ & $11.72 \pm 1.32$ & $11.00 \pm 1.25$ & $11.07 \pm 1.97$ & $9.44 \pm 2.00$ & $11.76 \pm 1.93$ & $14.33 \pm 2.05$ & $12.32 \pm 2.88$ & $9.24 \pm 2.44$ \\
\hline \multicolumn{9}{|l|}{ FUNCTIONAL: } \\
\hline $\mathrm{V}_{\mathrm{RBC}}(\mu \mathrm{m} / \mathrm{s})$ & $558.2 \pm 29.70$ & $517.28 \pm 27.3$ & $770.9 \pm 44.8$ & $638.8 \pm 42.9$ & $432.4 \pm 40.8$ & $404.9 \pm 44.4$ & $471.1 \pm 65.3$ & $508.2 \pm 53.7$ \\
\hline $\mathrm{f}_{\mathrm{RBC}}(\mathrm{RBC} / \mathrm{s})$ & $163.4 \pm 17.1$ & $177.6 \pm 16.2$ & $243.8 \pm 25.4$ & $168.1 \pm 25.8$ & $77.56 \pm 24.8$ & $51.84 \pm 26.4$ & $168.9 \pm 37.0$ & $312.9 \pm 31.4$ \\
\hline$H_{t}(\%)$ & $20.3 \pm 1.42$ & $21.5 \pm 1.26$ & $19.5 \pm 2.05$ & $18.0 \pm 1.93$ & $17.7 \pm 1.88$ & $18.3 \pm 2.02$ & $23.7 \pm 3.23$ & $28.0 \pm 2.52$ \\
\hline $\mathrm{L}_{\mathrm{D}}(\mathrm{RBC} / \mu \mathrm{m})$ & $0.328 \pm 0.034$ & $0.378 \pm 0.031$ & $0.258 \pm 0.051$ & $0.226 \pm 0.048$ & $0.186 \pm 0.046$ & $0.159 \pm 0.050$ & $0.541 \pm 0.074$ & $0.750 \pm 0.061$ \\
\hline $\mathrm{V}_{\mathrm{RBC}} \mathrm{H}_{\mathrm{t}}(\mathrm{RBC} \mu \mathrm{m} / \mathrm{s})$ & $138.2 \pm 15.7$ & $108.8 \pm 13.7$ & $215.8 \pm 22.2$ & $111.2 \pm 21.6$ & $105.6 \pm 20.5$ & $70.84 \pm 22.2$ & $93.12 \pm 36.2$ & $144.4 \pm 27.0$ \\
\hline$\tau_{\mathrm{W}}\left(\mathrm{N} / \mathrm{m}^{2}\right)$ & $0.475 \pm 0.031$ & $0.420 \pm 0.028$ & $0.648 \pm 0.045$ & $0.473 \pm 0.042$ & $0.363 \pm 0.041$ & $0.343 \pm 0.045$ & $0.416 \pm 0.071$ & $0.444 \pm 0.055$ \\
\hline \multicolumn{3}{|c|}{ Significant Difference between CN and IR } & \multicolumn{6}{|c|}{ Data are presented as Mean \pm SEM, $N=5$ animals, and $n=334$ vessels } \\
\hline $\mathrm{P}<0.01$ & & & $=0.05$ & & Significant Dif & erence betweer & $\mathrm{CN}$ and IR & \\
\hline
\end{tabular}


Table 4-3

The Interactions of Vessel Type and Radiation Status for 120 Days Post-Irradiation

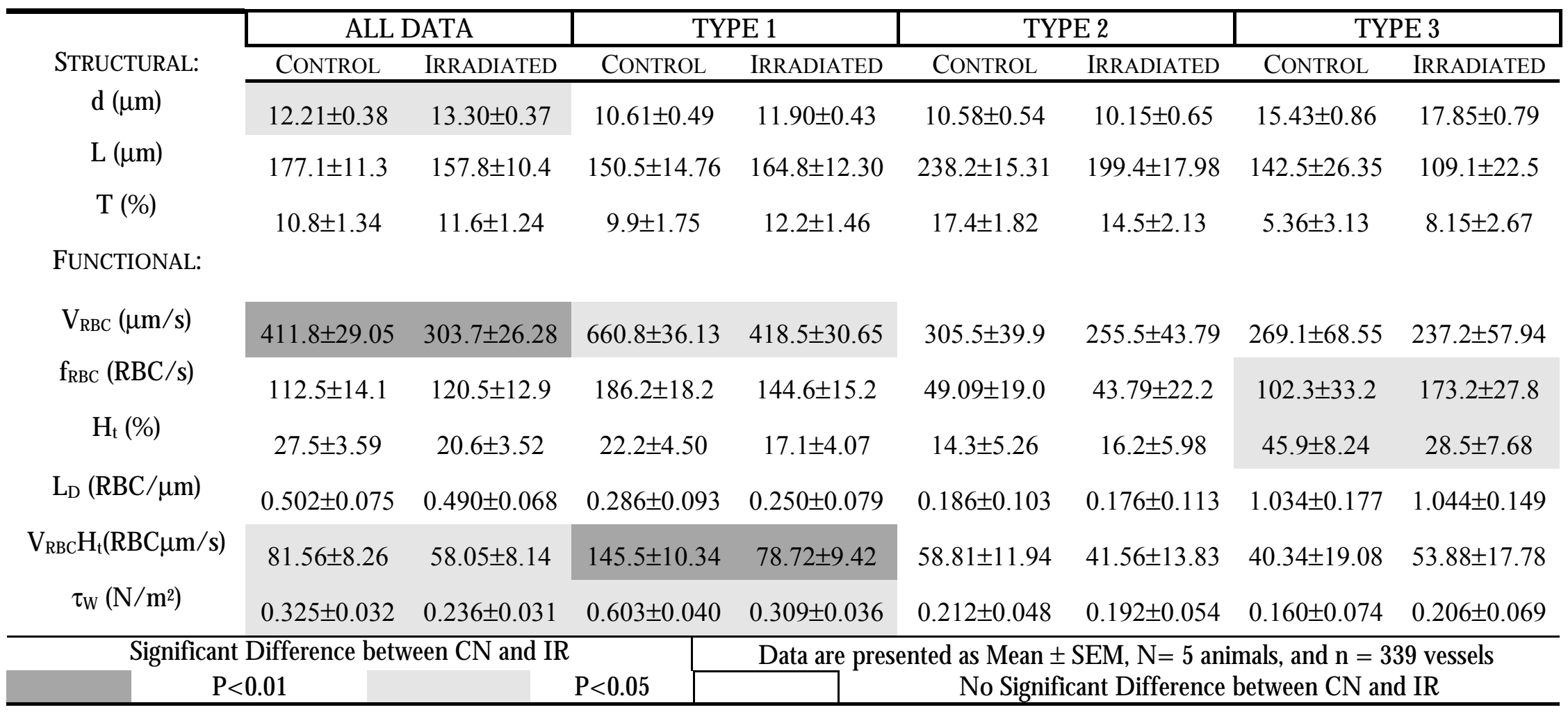


Table 4-4

The Interactions of Vessel Type and Radiation Status for 180 Days Post-Irradiation

\begin{tabular}{|c|c|c|c|c|c|c|c|c|}
\hline \multirow{3}{*}{$\begin{array}{l}\text { STRUCTURAL: } \\
\text { d ( } \mu \mathrm{m})\end{array}$} & \multicolumn{2}{|c|}{ ALL DATA } & \multicolumn{2}{|c|}{ TYPE 1} & \multicolumn{2}{|c|}{ TYPE 2} & \multicolumn{2}{|c|}{ TYPE 3} \\
\hline & CONTROL & IRRADIATED & CONTROL & IRRADIATED & CONTROL & IRRADIATED & CONTROL & IRRADIATED \\
\hline & $13.07 \pm 0.46$ & $13.99 \pm 0.42$ & \multirow{2}{*}{$\begin{array}{c}13.07 \pm 0.53 \\
177.7 \pm 16.41\end{array}$} & \multirow{2}{*}{$\begin{array}{c}13.29 \pm 0.59 \\
146.8 \pm 16.71\end{array}$} & \multirow{2}{*}{$\begin{array}{c}10.92 \pm 0.68 \\
284.5 \pm 20.46\end{array}$} & \multirow{2}{*}{$\begin{array}{c}11.10 \pm 0.69 \\
262.4 \pm 18.17\end{array}$} & $15.21 \pm 1.08$ & $17.58 \pm 0.90$ \\
\hline $\mathrm{L}(\mu \mathrm{m})$ & $221.6 \pm 13.02$ & $185.5 \pm 11.59$ & & & & & $202.7 \pm 28.93$ & $147.3 \pm 24.52$ \\
\hline $\mathrm{T}(\%)$ & $16.30 \pm 1.49$ & $14.97 \pm 1.32$ & $8.50 \pm 1.88$ & $12.97 \pm 1.91$ & $19.35 \pm 2.34$ & $19.31 \pm 2.08$ & $21.05 \pm 3.31$ & $12.62 \pm 2.80$ \\
\hline \multicolumn{9}{|l|}{ FUNCTIONAL: } \\
\hline $\mathrm{V}_{\mathrm{RBC}}(\mu \mathrm{m} / \mathrm{s})$ & $270.2 \pm 22.43$ & $476.9 \pm 20.92$ & $337.2 \pm 29.82$ & $579.2 \pm 29.39$ & $232.2 \pm 33.46$ & $426.8 \pm 32.57$ & $241.3 \pm 50.19$ & $424.6 \pm 44.89$ \\
\hline $\mathrm{f}_{\mathrm{RBC}}(\mathrm{RBC} / \mathrm{s})$ & $127.7 \pm 18.09$ & $197.6 \pm 17.12$ & $214.0 \pm 22.59$ & $275.4 \pm 23.85$ & \multirow{2}{*}{$\begin{array}{c}48.34 \pm 28.15 \\
21.2 \pm 3.51\end{array}$} & \multirow{2}{*}{$\begin{array}{c}77.05 \pm 27.66 \\
18.2 \pm 3.51\end{array}$} & $120.9 \pm 40.54$ & $240.5 \pm 36.13$ \\
\hline $\mathrm{H}_{\mathrm{t}}(\%)$ & $29.9 \pm 2.55$ & $22.2 \pm 2.22$ & $35.9 \pm 2.97$ & $28.9 \pm 3.16$ & & & $32.7 \pm 5.95$ & $19.5 \pm 4.70$ \\
\hline $\mathrm{L}_{\mathrm{D}}(\mathrm{RBC} / \mu \mathrm{m})$ & $0.508 \pm 0.039$ & $0.429 \pm 0.036$ & $0.631 \pm 0.052$ & $0.477 \pm 0.051$ & $0.285 \pm 0.058$ & $0.207 \pm 0.057$ & $0.607 \pm 0.088$ & $0.603 \pm 0.078$ \\
\hline $\mathrm{V}_{\mathrm{RBC}} \mathrm{H}_{\mathrm{t}}(\mathrm{RBC} \mu \mathrm{m} / \mathrm{s})$ & $63.88 \pm 9.51$ & $111.2 \pm 8.35$ & $101.4 \pm 11.28$ & $190.0 \pm 11.67$ & $40.56 \pm 13.32$ & $73.58 \pm 13.17$ & $49.66 \pm 22.56$ & $69.92 \pm 17.84$ \\
\hline$\tau_{\mathrm{w}}\left(\mathrm{N} / \mathrm{m}^{2}\right)$ & $0.310 \pm 0.043$ & $0.397 \pm 0.038$ & $0.417 \pm 0.052$ & $0.672 \pm 0.055$ & $0.204 \pm 0.061$ & $0.29 \pm 0.061$ & $0.31 \pm 0.104$ & $0.23 \pm 0.082$ \\
\hline Significant & Difference bet & veen $\mathrm{CN}$ and IF & & Data are $p$ & as IVle & $\mathrm{EM}, \mathrm{N}=5$ & als, and n = & vessels \\
\hline & 01 & & $\mathrm{P}<0.05$ & & No S & ant Differe & between CN & IR \\
\hline
\end{tabular}




\section{Table 4-5}

The Interactions of Time Post-Irradiation and Radiation Status for Type 1 Vessels

\begin{tabular}{|c|c|c|c|c|c|c|c|c|}
\hline \multirow{3}{*}{$\begin{array}{l}\text { STRUCTURAL: } \\
\text { d ( } \mu \mathrm{m})\end{array}$} & \multicolumn{2}{|c|}{ ALL DATA } & \multicolumn{2}{|c|}{ 60 DAYS } & \multicolumn{2}{|c|}{ 120 DAYS } & \multicolumn{2}{|c|}{180 DAYS } \\
\hline & CONTROL & IRRADIATED & CONTROL & IRRADIATED & CONTROL & IRRADIATED & CONTROL & IRRADIATED \\
\hline & $11.48 \pm 0.22$ & $11.97 \pm 0.22$ & $10.77 \pm 0.40$ & $10.71 \pm 0.40$ & $10.61 \pm 0.36$ & $11.90 \pm 0.32$ & $13.07 \pm 0.37$ & $13.29 \pm 0.41$ \\
\hline $\mathrm{L}(\mu \mathrm{m})$ & $151.6 \pm 8.74$ & $142.6 \pm 8.42$ & $126.5 \pm 16.49$ & $116.3 \pm 16.75$ & $150.5 \pm 14.45$ & $164.7 \pm 12.03$ & $177.7 \pm 14.36$ & $146.8 \pm 14.62$ \\
\hline $\mathrm{T}(\%)$ & $9.83 \pm 1.01$ & $11.55 \pm 0.97$ & $11.07 \pm 1.90$ & $9.43 \pm 1.93$ & $9.92 \pm 1.66$ & $12.25 \pm 1.39$ & $8.50 \pm 1.66$ & $12.97 \pm 1.69$ \\
\hline \multicolumn{9}{|l|}{ FUNCTIONAL: } \\
\hline $\mathrm{V}_{\mathrm{RBC}}(\mu \mathrm{m} / \mathrm{s})$ & $589.6 \pm 26.10$ & $545.5 \pm 24.42$ & $770.9 \pm 49.38$ & $638.7 \pm 47.20$ & $660.7 \pm 42.37$ & $418.4 \pm 35.95$ & $337.2 \pm 43.59$ & $579.2 \pm 42.97$ \\
\hline $\mathrm{f}_{\mathrm{RBC}}(\mathrm{RBC} / \mathrm{s})$ & $214.6 \pm 15.47$ & $196.0 \pm 15.11$ & $243.7 \pm 29.21$ & $168.0 \pm 29.66$ & $186.1 \pm 25.59$ & $144.6 \pm 21.31$ & $214.0 \pm 25.44$ & $275.4 \pm 26.87$ \\
\hline $\mathrm{H}_{\mathrm{t}}(\%)$ & $25.8 \pm 1.29$ & $21.3 \pm 1.26$ & $19.5 \pm 2.42$ & $18.0 \pm 2.29$ & $22.1 \pm 2.09$ & $17.1 \pm 1.89$ & $35.9 \pm 2.21$ & $28.9 \pm 2.35$ \\
\hline $\mathrm{L}_{\mathrm{D}}(\mathrm{RBC} / \mu \mathrm{m})$ & $0.391 \pm 0.023$ & $0.317 \pm 0.022$ & $0.258 \pm 0.044$ & $0.226 \pm 0.042$ & $0.285 \pm 0.038$ & $0.250 \pm 0.032$ & $0.631 \pm 0.039$ & $0.477 \pm 0.038$ \\
\hline $\mathrm{V}_{\mathrm{RBC}} \mathrm{H}_{\mathrm{t}}(\mathrm{RBC} \mu \mathrm{m} / \mathrm{s})$ & $154.2 \pm 10.70$ & $126.6 \pm 10.45$ & $215.7 \pm 19.68$ & $111.2 \pm 19.14$ & $145.5 \pm 17.37$ & $78.72 \pm 15.82$ & $101.4 \pm 18.49$ & $190.0 \pm 19.14$ \\
\hline$\tau_{\mathrm{W}}\left(\mathrm{N} / \mathrm{m}^{2}\right)$ & $0.555 \pm 0.034$ & $0.485 \pm 0.033$ & $0.647 \pm 0.064$ & $0.472 \pm 0.061$ & $0.602 \pm 0.055$ & $0.309 \pm 0.050$ & $0.417 \pm 0.059$ & $0.672 \pm 0.062$ \\
\hline \multicolumn{3}{|c|}{ Significant Difference between CN and IR } & & \multicolumn{5}{|c|}{ Data are presented as Mean \pm SEM, $N=5$ animals, and $n=511$ vessels } \\
\hline $\mathrm{P}<0.01$ & & & 0.05 & & Simificm Diff & mentum & $\mathrm{CN}$ and IR & \\
\hline
\end{tabular}




\section{Table 4-6}

The Interactions of Time Post-Irradiation and Radiation Status for Type 2 Vessels

\begin{tabular}{|c|c|c|c|c|c|c|c|c|}
\hline \multirow{3}{*}{$\begin{array}{l}\text { STRUCTURAL: } \\
\text { d ( } \mu \mathrm{m})\end{array}$} & \multicolumn{2}{|c|}{ ALL DATA } & \multicolumn{2}{|c|}{ 60 DAYS } & \multicolumn{2}{|c|}{ 120 DAYS } & \multicolumn{2}{|c|}{180 DAYS } \\
\hline & CONTROL & IRRADIATED & CONTROL & IRRADIATED & CONTROL & IRRADIATED & CONTROL & IRRADIATED \\
\hline & $10.27 \pm 0.18$ & $10.12 \pm 0.19$ & $9.32 \pm 0.29$ & $9.10 \pm 0.31$ & $10.57 \pm 0.29$ & $10.15 \pm 0.35$ & $10.92 \pm 0.35$ & $11.10 \pm 0.35$ \\
\hline $\mathrm{L}(\mu \mathrm{m})$ & $249.6 \pm 11.51$ & $241.3 \pm 11.88$ & $226.3 \pm 19.64$ & $262.2 \pm 20.89$ & $238.2 \pm 18.24$ & $199.4 \pm 21.42$ & $284.5 \pm 21.80$ & $262.4 \pm 19.36$ \\
\hline $\mathrm{T}(\%)$ & $16.16 \pm 1.32$ & $16.06 \pm 1.36$ & $11.76 \pm 2.26$ & $14.32 \pm 2.40$ & $17.37 \pm 2.10$ & $14.54 \pm 2.47$ & $19.35 \pm 2.51$ & $19.31 \pm 2.23$ \\
\hline \multicolumn{9}{|l|}{ FUNCTIONAL: } \\
\hline $\mathrm{V}_{\mathrm{RBC}}(\mu \mathrm{m} / \mathrm{s})$ & $323.3 \pm 17.45$ & $362.4 \pm 18.35$ & $432.4 \pm 28.96$ & $404.9 \pm 31.52$ & $305.5 \pm 30.16$ & $255.4 \pm 33.09$ & $232.2 \pm 31.52$ & $426.8 \pm 30.68$ \\
\hline $\mathrm{f}_{\mathrm{RBC}}(\mathrm{RBC} / \mathrm{s})$ & $58.3 \pm 3.62$ & $57.5 \pm 3.85$ & $77.5 \pm 6.17$ & $51.8 \pm 6.56$ & $49.0 \pm 5.77$ & $43.7 \pm 6.73$ & $48.3 \pm 6.85$ & $77.0 \pm 6.73$ \\
\hline$H_{t}(\%)$ & $17.7 \pm 1.15$ & $17.5 \pm 1.23$ & $17.7 \pm 1.83$ & $18.3 \pm 1.97$ & $14.3 \pm 2.01$ & $16.2 \pm 2.28$ & $21.1 \pm 2.14$ & $18.1 \pm 2.14$ \\
\hline $\mathrm{L}_{\mathrm{D}}(\mathrm{RBC} / \mu \mathrm{m})$ & $0.219 \pm 0.013$ & $0.180 \pm 0.013$ & $0.185 \pm 0.021$ & $0.159 \pm 0.023$ & $0.186 \pm 0.022$ & $0.175 \pm 0.025$ & $0.285 \pm 0.023$ & $0.207 \pm 0.023$ \\
\hline $\mathrm{V}_{\mathrm{RBC}} \mathrm{H}_{\mathrm{t}}(\mathrm{RBC} \mu \mathrm{m} / \mathrm{s})$ & $68.3 \pm 8.07$ & $61.9 \pm 8.66$ & $105.6 \pm 12.66$ & $70.8 \pm 13.69$ & $58.8 \pm 13.96$ & $41.56 \pm 16.17$ & $40.5 \pm 15.20$ & $73.5 \pm 15.03$ \\
\hline$\tau_{\mathrm{W}}\left(\mathrm{N} / \mathrm{m}^{2}\right)$ & $0.259 \pm 0.016$ & $0.275 \pm 0.017$ & $0.362 \pm 0.025$ & $0.343 \pm 0.028$ & $0.212 \pm 0.028$ & $0.192 \pm 0.031$ & $0.204 \pm 0.030$ & $0.29 \pm 0.03$ \\
\hline \multicolumn{4}{|c|}{ Significant Difference between CN and IR } & \multicolumn{5}{|c|}{ Data are presented as Mean \pm SEM, $N=5$ animals, and $n=395$ vessels } \\
\hline \multicolumn{2}{|c|}{$\mathrm{P}<0.01$} & \multicolumn{2}{|c|}{$\mathrm{P}<0.05$} & & \multicolumn{4}{|c|}{ No Significant Difference between CN and IR } \\
\hline
\end{tabular}


Table 4-7

The Interactions of Time Post-Irradiation and Radiation Status for Type 3 Vessels

\begin{tabular}{|c|c|c|c|c|c|c|c|c|}
\hline \multirow{3}{*}{$\begin{array}{l}\text { STRUCTURAL: } \\
\mathrm{d}(\mu \mathrm{m})\end{array}$} & \multicolumn{2}{|c|}{ ALL DATA } & \multicolumn{2}{|c|}{ 60 DAYS } & \multicolumn{2}{|c|}{120 DAYS } & \multicolumn{2}{|c|}{180 DAYS } \\
\hline & CONTROL & IRRADIATED & CONTROL & IRRADIATED & CONTROL & IRRADIATED & CONTROL & IRRADIATED \\
\hline & $14.39 \pm 1.03$ & $17.03 \pm 0.89$ & $12.53 \pm 1.66$ & $15.66 \pm 1.43$ & $15.43 \pm 1.70$ & $17.85 \pm 1.55$ & $15.21 \pm 2.00$ & $17.58 \pm 1.66$ \\
\hline $\mathrm{L}(\mu \mathrm{m})$ & $156.7 \pm 11.54$ & $121.3 \pm 9.81$ & $125.0 \pm 19.19$ & $107.5 \pm 16.29$ & $142.4 \pm 20.56$ & $109.1 \pm 17.57$ & $202.7 \pm 20.19$ & $147.3 \pm 17.11$ \\
\hline $\mathrm{T}(\%)$ & $12.91 \pm 1.59$ & $10.00 \pm 1.35$ & $12.32 \pm 2.65$ & $9.23 \pm 2.25$ & $5.36 \pm 2.84$ & $8.15 \pm 2.42$ & $21.05 \pm 2.79$ & $12.62 \pm 2.36$ \\
\hline \multicolumn{9}{|l|}{ FUNCTIONAL: } \\
\hline $\mathrm{V}_{\mathrm{RBC}}(\mu \mathrm{m} / \mathrm{s})$ & $327.2 \pm 25.78$ & $389.9 \pm 22.02$ & $471.1 \pm 42.64$ & $508.1 \pm 35.05$ & $269.1 \pm 47.67$ & $237.1 \pm 40.29$ & $241.3 \pm 43.52$ & $424.6 \pm 38.92$ \\
\hline $\mathrm{f}_{\mathrm{RBC}}(\mathrm{RBC} / \mathrm{s})$ & $130.7 \pm 26.46$ & $242.2 \pm 22.76$ & $168.8 \pm 43.44$ & $312.8 \pm 36.88$ & $102.3 \pm 47.43$ & $173.2 \pm 39.76$ & $120.9 \pm 46.55$ & $240.5 \pm 41.48$ \\
\hline $\mathrm{H}_{\mathrm{t}}(\%)$ & $34.1 \pm 7.46$ & $25.3 \pm 6.21$ & $23.6 \pm 12.26$ & $28.0 \pm 9.54$ & $45.9 \pm 12.26$ & $28.5 \pm 11.4$ & $32.7 \pm 14.16$ & $19.5 \pm 11.19$ \\
\hline $\mathrm{L}_{\mathrm{D}}(\mathrm{RBC} / \mu \mathrm{m})$ & $0.727 \pm 0.149$ & $0.799 \pm 0.128$ & $0.540 \pm 0.247$ & $0.749 \pm 0.203$ & $1.034 \pm 0.277$ & $1.044 \pm 0.234$ & $0.607 \pm 0.277$ & $0.603 \pm 0.226$ \\
\hline $\mathrm{V}_{\mathrm{RBC}} \mathrm{H}_{\mathrm{t}}(\mathrm{RBC} \mu \mathrm{m} / \mathrm{s})$ & $61.0 \pm 8.97$ & $89.3 \pm 7.38$ & $93.1 \pm 14.74$ & $144.3 \pm 10.99$ & $40.34 \pm 14.74$ & $53.88 \pm 13.75$ & $49.66 \pm 17.02$ & $69.9 \pm 13.46$ \\
\hline$\tau_{\mathrm{W}}\left(\mathrm{N} / \mathrm{m}^{2}\right)$ & $0.295 \pm 0.033$ & $0.293 \pm 0.028$ & $0.416 \pm 0.055$ & $0.443 \pm 0.043$ & $0.160 \pm 0.055$ & $0.206 \pm 0.051$ & $0.310 \pm 0.063$ & $0.230 \pm 0.050$ \\
\hline \multicolumn{4}{|c|}{ Significant Difference between CN and IR } & \multicolumn{5}{|c|}{ Data are presented as Mean \pm SEM, $N=5$ animals, and $n=205$ vessels } \\
\hline \multicolumn{2}{|c|}{$\mathrm{P}<0.01$} & \multicolumn{2}{|c|}{$\mathrm{P}<0.05$} & & \multicolumn{4}{|c|}{ No Significant Difference between CN and IR } \\
\hline
\end{tabular}




\section{Chapter 5. DISCUSSION}

Therapeutic doses of ionizing radiation result in changes in the structure and function of microvascular networks in normal tissue. The late effects of ionizing radiation $(60,120$, and 180 days post-irradiation) on microvascular networks were studied in a hamster cremaster muscle model. The work complemented an earlier study in our lab which examined the early effects of ionizing radiation ( 3,7 , and 30 days post-irradiation) on microvascular networks [1-3]. The set of protocols developed to study the early effects of irradiation was also used to examine the late effects of ionizing radiation on normal tissue microvascular networks. The early effects study showed significant changes in the structural and functional parameters of microvascular networks. Likewise, our late effect study also indicated that ionizing radiation significantly alters the structure and function of microvascular networks. However, not all parameters that showed significant changes in the early effects study after irradiation were altered in the late effects study.

A complete microvascular networks was separated into different vessel types (i.e., type1, type 2, and type 3) according to the vessel's bifurcation ends. This method of classification was more informative than studying randomly selected vessels.

\section{Comparison of Control Data with Literature}

As in the early effects study of irradiation, we saw variability in the control data with age which is due to the normal process of aging. These changes in controls can be attributed to the normal process of maturation of microvascular networks. 
Reported studies of microvascular changes with age have not been extended beyond 100 days of age making comparison with other work difficult. In the current study we observed changes in control animals up to 230 days of age.

In our comparison, only type 2 vessels (one diverging and one converging bifurcation) will be discussed to compare with "capillary" vessels often reported in literature. In addition, the comparison below were not made with aged match animals. The average length of "capillaries" has been reported as $222 \pm 7.1 \mu \mathrm{m}$ [31], while our result indicated type 2 vessel length of $186 \pm 6.8$ $\mu \mathrm{m}$. Our early effects study had length of $199 \pm 10.7 \mu \mathrm{m}[1]$. Average RBC velocity has been indicated to range from 42 to $545 \mu \mathrm{m} / \mathrm{s}$ with an average of $152 \mu \mathrm{m} / \mathrm{s}$ [32], while our results suggest $\mathrm{RBC}$ velocity in type 2 velocity to be $413.4 \pm 15.7 \mu \mathrm{m} / \mathrm{s}$, and our early effects study had velocity of $302 \pm 14 \mu \mathrm{m} / \mathrm{s}[1]$. Vessel hematocrit has been reported in the range of 1.2 to $32.5 \%$ with an average of $13.3 \pm 5.7 \%$ [32], while we calculated a vessel hematocrit of $25.9 \pm 1.5 \%$. Vessel diameters were reported to range from 4 to $27 \mu \mathrm{m}$ for arterioles, capillaries, and venules [33]. Our studies show vessel diameters to range from 8.42 to $17.7 \mu \mathrm{m}$ for all vessel types. Thus, control data from our late effect study are in general agreement with data from our earlier study and previously published works.

\section{Effects of Irradiation}

Consistent with our early effects study, the body weight of irradiated animals was significantly less than controls at 60 and 120 days post-irradiation. However, at 180 days postirradiation the weight of irradiated animals was not significantly different from controls (see figure 5-1). In the clinical environment, radiation exposure is known to cause a decrease in body 
weight. Therefore, because the testicle region of hamster comprises $20 \%$ of the animal's body, a significant drop in body weight at 60 and 120 days post-irradiation seems logical. However, at 180 days post-irradiation, the animal's weight appears to have recovered from the radiation exposure. Another possible explanation could be that at 180 days the gain of weight due to aging is much greater than the late effects of radiation exposure.

Arteriolar tone in irradiated animals was not significantly different from control for all time post-irradiation. In our early effect study, we saw significant changes only at 3 days post irradiation and attributed the change to an early response to radiation, possibly affecting the sensitivity of adenosine receptors [1]. Hence, the current data for the late effects was in agreement with our earlier study.

Average RBC transit time showed no significant differences between irradiated and controls. This finding also coincides with our early effects study in which we observed no significant difference in RBC transit time between irradiated and control animals.

The diameter of irradiated vessels was greater as compared to control for all late time post-irradiation and significantly greater than controls at 120 days post-irradiation. Data also suggested that type 3 irradiated vessels were significantly greater than controls for all time postirradiation. We therefore concluded that radiation affected type 3 vessels more than type 1 and type 2 vessels. The increase in vessel diameter at all time post-irradiation may be due to the excessive endothelial regeneration in vessel in response to the acute effect of radiation. It has been documented that immediately after irradiation there is loss of endothelial and smooth muscle cells [20]. The loss in endothelial and smooth muscle cells can be used to explain the decrease in vessel diameter due to irradiation that our lab observed in the early effects study. As the tissue regenerates from the radiation damage, excessive endothelial cells and smooth muscle 
cells regeneration can lead to an increase in vessel diameter [20]. This is consistent with our findings in both early and late effects studies. As for the latter point, which states that radiation affects type 3 vessels more than other vessels, the data was in general agreement with our early effect study [1]. Like our early effects study, this finding could be attributed to normal tissue maturation and suggests that ionizing radiation alters this maturation process [1].

The tortuosity of vessels was found not to be significantly different between control and irradiated animals at any time points. However, our early-effect study observed irradiated vessels to be more tortuous than controls at 7 days post-irradiation. These data differed from published work, which suggested irradiation leads to ischemic environment that stimulate endothelial proliferation causing the small veins to become elongated and tortuous [34].

While average $\mathrm{RBC}$ velocity decreased with age in control animals, the average RBC velocity in irradiated vessels showed a decrease from controls at 120 days post-irradiation and then an increase at 180 days post-irradiation (see figure 4-3). At 120 days post-irradiation only the $\mathrm{RBC}$ velocity in type 1 vessels was significantly smaller as compared to control. Whereas, at 180 days post-irradiation, the increase in velocity from controls was present in all vessel types. Like our early effect studies, changes in RBC velocity could not be correlated with changes in vessel diameter.

Significant changes in the RBC flux of irradiated vessels from controls were found only at 180 days post-irradiation. Here, irradiated animals had higher RBC flux in all vessel types. Data also showed that type 3 vessels had higher RBC flux in irradiated networks at all time points. This was in agreement with published work which suggested that loss and occlusion of capillaries due to irradiation can result in shunting of arterial blood to the venules [35]. 
Furthermore, there was no correlation between vessel diameter and RBC flux although both have similar trends.

The vessel hematocrit of control networks increases over time while the vessel hematocrit of irradiated networks was unchanged at all time points (see figure 5-4). The vessel hematocrit of irradiated networks was significantly less than controls at 120 and 180 days post-irradiation. However, these results differ from our early time points that found a significant increase in vessel hematocrit of irradiated network at both 7 and 30 days post-irradiation.

At 60 and 120 days post-irradiation, vessel wall shear stress in type 1 irradiated vessels was significantly smaller than controls while at 180 days post-irradiation, vessel wall shear stress in type 1 irradiated vessels was significantly greater than controls. Vessel wall shear stress has been shown to cause changes in the rolling and adhering of leukocytes in venules. However, our data found significant changes in only type 1 vessels and not type 3 suggesting that rolling and adhering of leukocytes may not be significantly affected. This contradicts published work and thus more research needs to be perform in this area. In addition, it has been hypothesized that microvascular network remodeling is regulated by mechanical stress (i.e., vessel wall shear stress) [36]. In particular, changes in vessel diameter are often correlated with changes in vessel shear stress (see Equation 4-15). However, in the current study, we did not observe a correlation between vessel diameter and wall shear stress. Therefore, vessel wall shear stress may not play an important role in remodeling after irradiation since data showed not difference from controls. 


\section{Chapter 6. CONCLUSION}

1) Therapeutic doses of ionizing radiation significantly alter the structure and function of normal tissue microvascular networks at 60, 120, and 180 days post-irradiation.

2) Studying complete microvascular networks is more informative than just randomly selected vessels.

3) Therapeutic doses of ionizing radiation interfere with the normal maturation process of the microvascular networks.

4) Type 3 vessels (post capillary venules) are the most sensitive to radiation.

The early effects and late effects studies have set a foundation for our study of how ionizing radiation affects normal tissue. We hope to continue the study by using the collected data to mathematically model the oxgenation of microvascular networks. We believe ionizing radiation might alter the oxygen delivery to the tissue. In addition, Prabhakar Pandian has begun to examine how ionizing radiation affects human endothelial cells, and Hong Yuan has begun to observe how ionizing radiation affects the blood brain barrier. 


\section{List of References}




\section{References}

1. Roth NM. Early effects of ionizing radiation on microvasculature. 1997. University of Tennessee, Memphis. 12-5-1997. Ref Type: Thesis/Dissertation.

2. Roth NM, Kiani MF. A "Geographic Information Systems" based method for the study of microvascular networks. Annals of Biomedical Engineering. 1999; 27(1): 42-47.

3. Roth NM, Sontag MR, Kiani MF. Early effects of ionizing radiation on normal tissue microvascular networks. Radiation Research. 1999; 151(3): 270-277.

4. Hendrickson FR, Withers HR. Principles of radiation oncology. American Cancer Society textbook of Clinical Oncology. Atlanta, GA: American Cancer Society, Inc. 1991; 35-36.

5. Wilson JF. Syllabus: a categorical course in radiation therapy: cure with preservation of function and aesthetics. Oak Brook, IL: Radiological Society of North America, Inc., 1988; 5-6.

6. Rubin P, Siemann DW. Principles of radiation oncology and cancer radiotherapy. Clinical Oncology. Rochester, NY: W.B. Saunders Co., 1993; 71-88.

7. Hall EJ. Radiobiology for the radiobiologist. Philadelphia: J.B. Lippincott, 1988.

8. Elkind MM. The radiobiology of cultured mammalian cells. New York, NY: Gordon \& Breach, 1967.

9. Silverberg E, Boring CC, Squires TS. Cancer statistics. American Cancer Society, Inc., 1990; 9-26.

10. Rubin P, Cooper RA. Clinical oncology. Rochester, NY: W.B. Saunders Co., 1993; 1-20.

11. Warburg OH. The metabolism of tumors: investigations from the Kaiser Wilhelm Institute for Biology. London, UK: Constable and CO., Ltd. 1930.

12. Bishop JM. The molecular genetics of cancer. Science. 1992; 1235: 308-311.

13. Hoover R, Fraumeni JF. Drug-induced cancer. Cancer. 1981; 47: 1071-1080.

14. Henshaw EC. The biology of cancer. Clinical Oncology. 1993; 23-27.

15. Tortora GJ, Grabowski SR. Principles of anatomy and physiology. 1996; 89-90.

16. Jain RK. Delivery of molecular medicine to solid tumors. Science. 1996; 271: 1079-1080.

17. Jain RK. Delivery of molecular and cellular medicine to solid tumors. Microcirculation 1997; 4: 1-23. 
18. Cancer Radiation Therapy. http://www.medlogic.com/ssO8.html 1999.

19. Rubin P, Nelson DF. Late effects of cancer treatments:radiation and drug toxicity. Clinical Oncology. Rochester: W.B. Saunders Co., 1999; 735-760.

20. Hendee WR. Proposition: long-term changes in irradiated tissues are due principally to vascular damage in the tissue. Medical Physiology 1998; 25: 2265-2268.

21. Krishnan EC, Krishnan L, Jewell B, Bhatia P, Jewell WR. Dose-dependent radiation effect on microvasculature and repair. Journal National Cancer Institute. 1987; 79(6) 1321-1325.

22. Hopewell JW. Early and late changes in the functional vascularity of the hamster cheek pouch after local irradiation. Radiation Research. 1975; 63: 157-164.

23. Wu NZ, Ross BA, Gulledge C, Klitzman B, Dodge R, Dewhirst MW. Differences in leucocyte-endothelium interactions between normal and adenocarcinoma bearing tissues in response to radiation. British Journal Cancer. 1994; 69(5): 883-889.

24. Gaehtgens P. Why networks? International Journal of Microcirculation Clinical Experience. 1992; 11: 123-132.

25. Fung YC. Biomechanics: circulation. New York: Springer-Verlag, 1999.

26. Chien S, Usami S, Skalak R. Blood flow in small tubes. Handbook of Physiology. Baltimore, MD: Waverly Press, 1984; 4: 217-249.

27. Fahraeus R, Lindqvist T. The viscosity of the blood in narrow capillary tubes. American Journal of Physiology. 1931; 96: 562-568.

28. Sweeney TE, Sarelius IH. Arteriolar control of capillary cell flow in striated muscle. Circulation Research. 1989; 64: 112-120.

29. Cokelet GR. Experimental determination of the average hematocrit of blood flowing in a vessel. Microvascular Research. 1974; 7: 382-384.

30. Pries AR, Secomb TW, Gaehtgens P, Gross JF. Blood flow in microvascular networks, experiments and simulation. Circulation Research. 1990; 67: 826-834.

31. Berg BR, Sarelius IH. Functional capillary organization in striated muscle. American Journal of Physiology. 1995; 268: H1215-H1222.

32. Klitzman B, Johnson PC. Capillary network geometry and red cell distribution in hamster cremaster muscle. American Journal of Physiology. 1982; 242: H211-H219.

33. Kiani MF, Cokelet GR, Sarelius IH. Effect of diameter variability along the capillary segment on apparent pressure drop. Fifth World Congress Microcirc. 1991; 50. 
34. Reinhold HS, Buisman GH. Repair of radiation damage to capillary endothelium. British Journal Radiology. 1975; 48: 727-731.

35. Tannock IF, Steiner M. Quantitative techniques for study of the anatomy and function of small blood vessels in tumors. Journal National Cancer Institute. 1969; 42: 771-782.

36. Skalak TC, Price RJ. The role of mechanical stresses in microvascular remodeling. Microcirculation. 1996; 3: 143-165. 


\section{List of Appendixes}




\section{Appendix A}

\section{Steps In Tracing a Network and Creating Topology}

1) Configuring drawing screen

Configure Calcomp DrawingBoard III to make sure the board drawing area fits on Autocad screen. If not, go under "Options" and then to "Tablet" and "Configure". Follow instructions.

2) Digitize setup

Under "Map", go to "Data entry", then "Digitize setup." Check "Linear" in Object Type box. The pre-setting should be Create on Layer= "0", Linetype= "Bylayer", Elevation= "2D", and Width= "0.00". Click "OK" if setting is correct.

3) Digitize Network

Under "Map", go to "Data entry", then "Digitize." Start tracing network using the Calcomp Digital Mouse which has 4 buttons numbered 0-3. Make sure to start at the feeding vessels and work your way down the network. Click the " 0 " button at every curvature for each vessel. Each time the "0" button is press will represent a blue grip in AutoCAD drawing. Once a vessel is drawn press the 1 button to begin a new vessel. Before pressing the 0 button to start a new vessel, press the " 2 " button, and select "endpoint" if you are beginning a new bifurcation or select "intersection" if you are finishing a bifurcation. This will cause the end grips of the vessels to overlap at nodes, thus connecting vessels together. When done, zoom in the drawing to make sure that all the end grips are connected at each bifurcation. This is very important because topology will not work, otherwise. To perform this task, zoom in and click on three vessels so that blue grips will appear. When all three vessels are connected, moving the common grip will cause animation in the three vessels. Lastly, using an image of a micrometer, draw a line 100 um near the network.

4) Create Topology Query

Under "Map", go to "Topology" and then to "Create." In the Define Topology box, name the topology as "Connections" and gives its description as "Vessel Connections". Select the type of topology as "Network". Under Topology Objects, select "Node Objects", and when an Node Object Box opens, select "Select Automatically" and "Create Node Objects". Click OK. Also under Topology Objects, select "Link Objects" and then select "Select Automatically." Click OK. Lastly, click "Proceed". If the topology was created successful, at the command prompt, it should read "topology successfully created" and the number of objects created.

5) Define Topology Query to Draw

Under "Map", go to "Define Topology Query". Under Name Query Topology, the default should read "Connections". Otherwise, select "Load" and another box will open which will allow you to select "Connections". After "Connections" has been set as the Topology Name, select "Define Query." A Define Query box should open. In the Query Type section, select "AND", and then select "Property." After the Property Condition box opens, select "Object Type" and then "Value." After Select box opens, select

"2DPOLYLINE" and then OK. Click OK again to close the Property Condition box. (This query only find 2Dpolylines, but since the lastest version of AutoCAD 14 save the lines as lightweight polylines, another query must be performed.) Next, in the Query Type section, 
select "OR" and then "Property". Again, select "Object Type" and then "Value". In the Select box, scroll down and select "LWPOLYLINE". Click OK and then OK again to get back to the Define Query box. In the Query Mode section, select "Draw", and in the Options section, select "Alter Properties..". Once the Set Alteration Properties box appears, select

"Text". A Define Text box will appear. Select "Data" and a Select Data box will appear. In "Tables:", scroll down and select the "TPMLINK Connections". This will allow you to select "ID" as the first Data Field. Click OK. The ID address is now in the "Text Value" section. Select "Color Palette" so you can change the color to blue. Perform the above procedure again to get "Start Node" and "End Node". The Node's heights is 0.15 and colors are red and magenta, repectively. After getting back to the Define Query box, select "Execute Query", and three seconds later the IDs and Nodes will be display on each vessels.

6) Editing Text Values

After text values have been generated, move ID values to their corresponding vessels. Node should be placed at start and end of vessels. Some start and end nodes will need to be deleted because nodes will overlap at bifurcation.

7) Define Topology Query to Report

Like above, set query type to "2DPOLYLINES" or "LWPOLYLINE". In the Query Mode, select "Report" and click on "Options". An Output Report Option box will appear. Select "Data", and then again choosing TPMLINK Connections table, select ID. Click OK and then select "Add" to add the address to the report template. Again do the same for Start and End Nodes. Next select "Properties" and scroll down and select "length". Click OK and select "Add" to add to report template. Perform this task again for object start point and object end point. Lastly, select "Browse in the Output Report Option" and select the name and directory of the text file. Click OK, and select "Execute Query." Using Windows Explorer, check the output file.

\section{How to Run Colwid.Isp to Change Diameter and Color of Vessels}

\section{1) Format of External Database}

Create an excel file with two columns. The first column contains normalized diameter values which can be real numbers, and the second column contains the color code which must be integer ranging from 0 to 256. The order of the values in the columns must start with the smallest ID number and go down to highest. The excel file must then be save as a text file in a specific directory that matches with the name of text file in the program.

2) Changing the name and location of the database in Colwid.lsp

Open Colwid.lsp in Notepad. The second line of text reads

$$
\text { (setq f (open "e: \|vinh \|test \test2.txt" "r")) }
$$

This means that the current location of the database is stored in "e:lvinhltestltest2.txt". If the file is saved elsewhere, only change the content of the first quotation. For example, if the file is now called "d:|program files lautocad \diamcolor.txt", then the second line in Colwid.lsp should read as

$$
\text { (setq f (open "e: \progam files \\autocad \diamcolor.txt" "r")) }
$$

Afterward, save the file as Colwid.lsp.

3) Executing the Routine 
Open the desire AutoCAD drawing. Zoom the drawing to "Extent" so that every object fits in the drawing screen. Turn on only the layer that contain the polylines of vessels. At the command prompt, type "appload". After a Load AutoLisp File box appears, select "File" to find colwid.lsp and then select "Load". The file is now loaded, type "colwid" at the command prompt to execute the program. The diameter and color of vessels should change instantly.

\section{Body of Text for Colwid.lsp}

(defun c:colwid()

(setq f (open "e: \|vinh\\test \test2.txt" "r"))

(setq ss1 (ssget "X" '((0 . "LWPOLYLINE"))))

(setq $\mathrm{n}$ (sslength ss 1$)$ )

(setq test 1)

(while $(>=\mathrm{n}$ test)

(princ test)

(setq d (read-line f))

$(\operatorname{setq} \mathrm{g}(\operatorname{read} \mathrm{d}))$

$($ setq $x(-$ test 1$))$

(setq en (ssname ss $1 \mathrm{x})$ )

(setq ed (entget en))

(setq ed

(subst (cons $43 \mathrm{~g})$ (assoc 43 ed) ed))

(entmod ed)

(setq test $(+$ test 1$))$

)

(close f)

( setq f (open "e: |lvinh \|test \test2.txt" "r"))

(setq test 1)

(while ( $>=\mathrm{n}$ test)

(princ test)

(setq first 100)

(while (/= first 10)

(setq a (read-char f))

(if (= a 9)

(progn

(princ test)

( setq $b($ read-char $\mathrm{f}))$

( $\operatorname{setq} \mathrm{c}(\operatorname{read}-\mathrm{char} \mathrm{f}))$ 


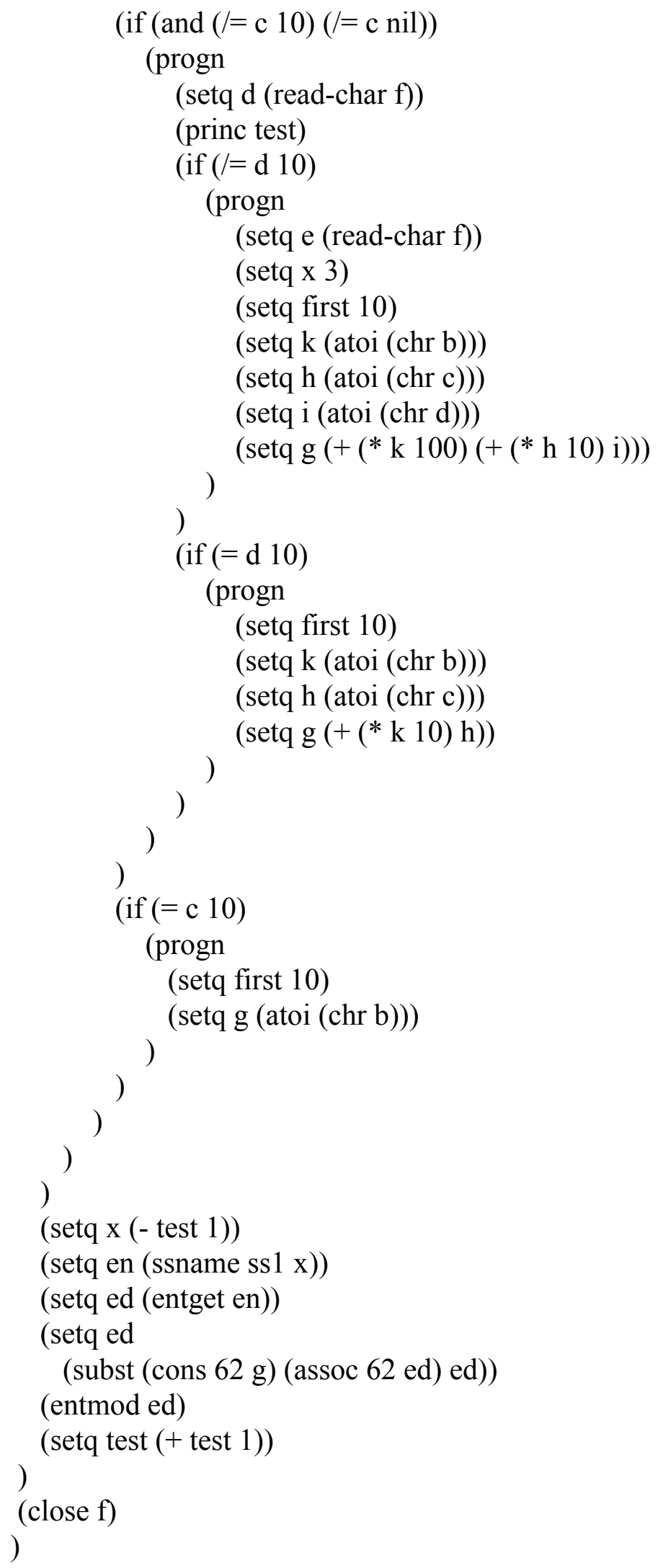




\section{Appendix B}

\section{Summary of Data for Early And Late Effects Studies.}

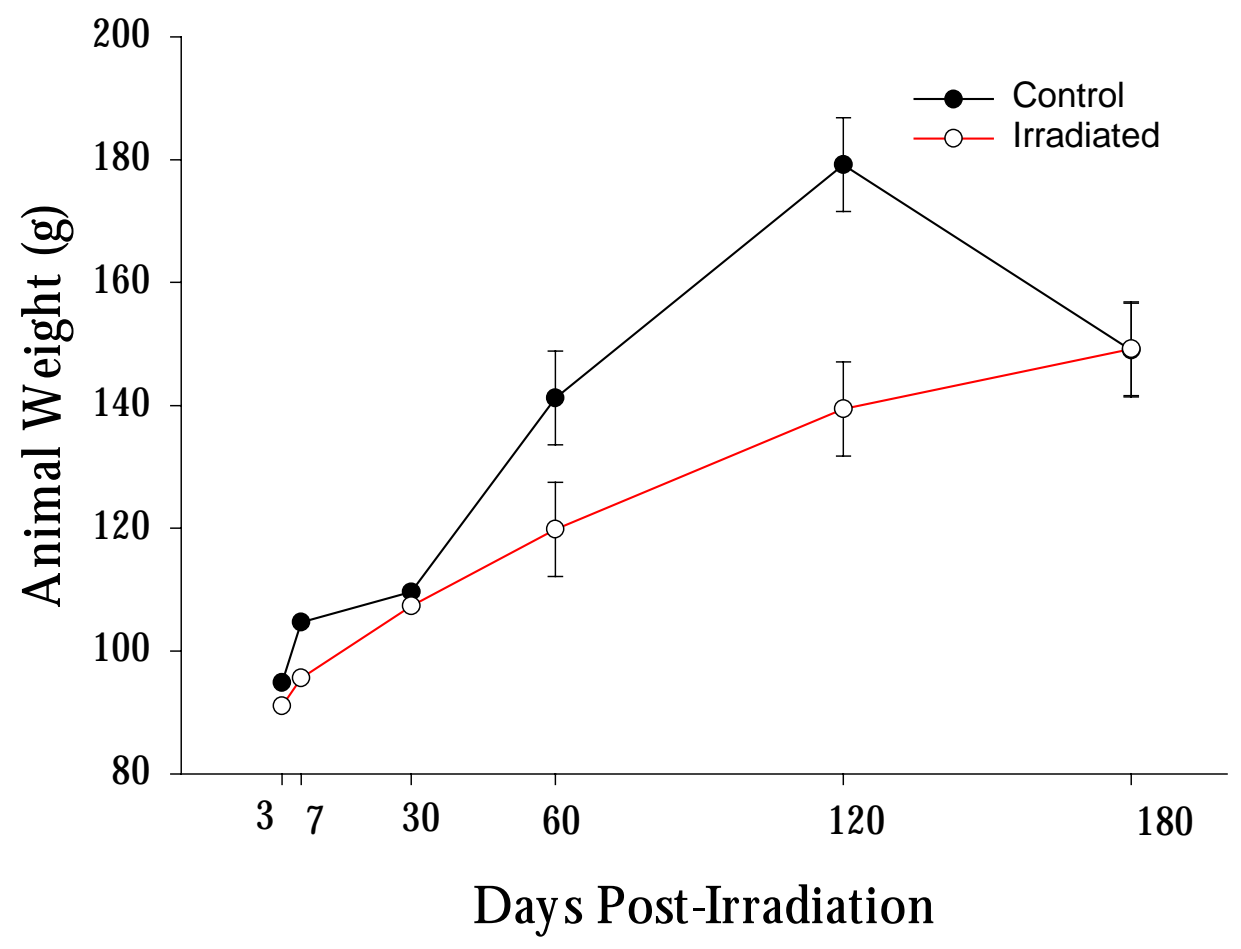

Figure B-1. Animal weight at 3, 7, 30, 60, 120, 180 days post-irradiation $(\mathrm{N}=5$ animals per time point per treatment group. Data are presented as Mean \pm SEM $(\mathrm{P}<0.05)$. 


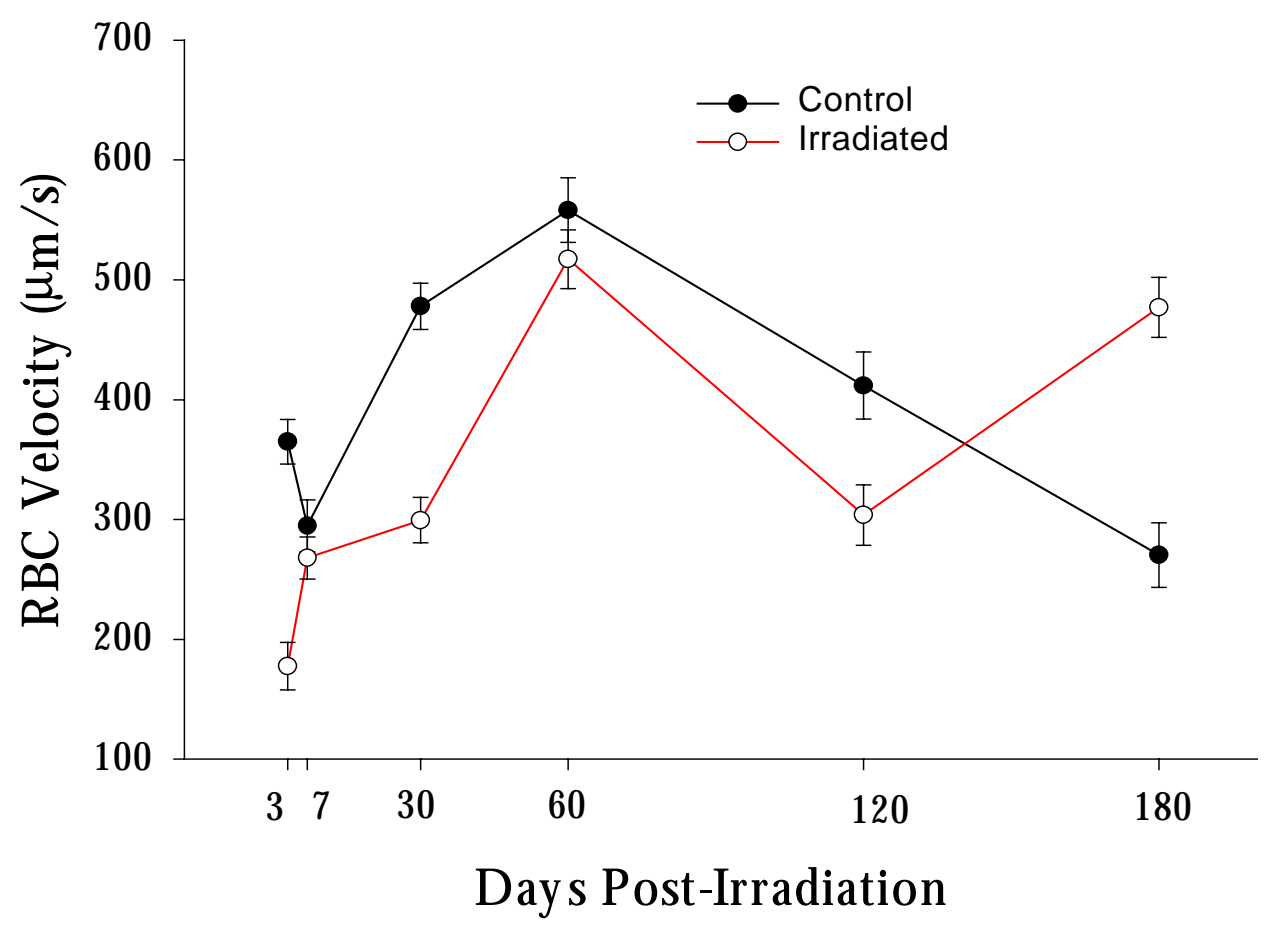

Figure B-2. RBC velocity at 3, 7, 30, 60, 120, 180 days postirradiation ( $\mathrm{N}=5$ animals per time point per treatment group). Data are presented Mean \pm SEM $(\mathrm{P}<0.05)$.

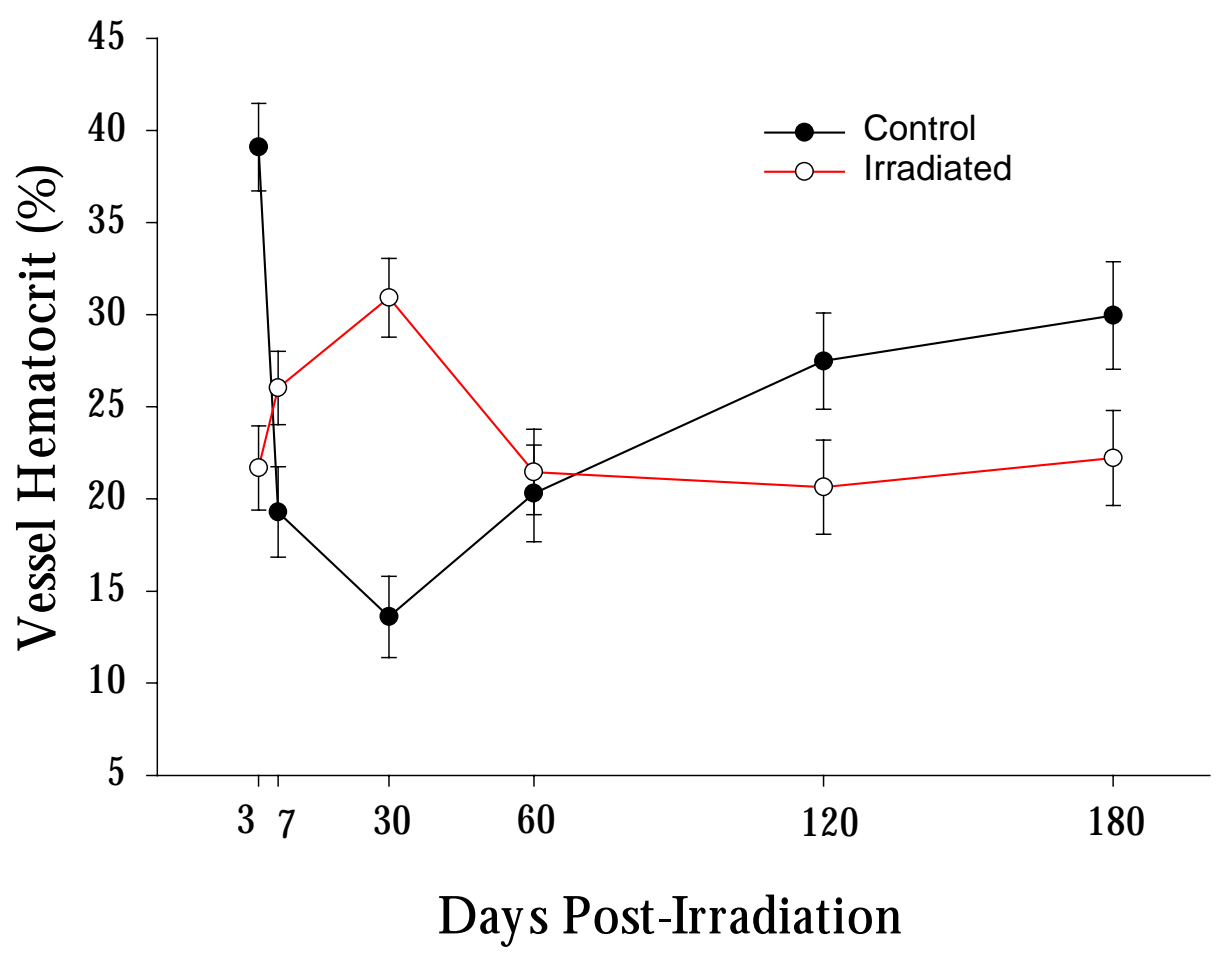

Figure B-3. Vessel hematocrit at 3, 7, 30, 60, 120, 180 days postirradiation ( $\mathrm{N}=5$ animals per time point per treatment group). Data are presented Mean \pm SEM $(\mathrm{P}<0.05)$. 


\section{VITA}

Vinh Nguyen was born on September 24, 1975, in Saigon, Vietnam. He immigrated to the United States in 1981 and has resided in New Orleans, Louisiana ever since. After graduating from Louisiana School for Math, Science \& the Arts high school where he majored in math and science, Vinh continued his education at Columbia University, School of Engineering, where he received a Bachelor of Science in Bioengineering in June 1996. While a student at Columbia, he participated in numerous extracurricular activities which included Psi Upsilon Fraternity, Student Government Council, Vietnamese Student Association. Upon graduation, Vinh decided to continue his education towards a Masters of Science in Biomedical Engineering at the University of Tennessee, Memphis, School of Biomedical Engineering where he was recruited to the laboratory of Mohammad F. Kiani, Ph.D., who served as his major advisor and mentor. While in Memphis, Vinh was awarded numerous travel awards to scientific conferences and participated in Student Government Association. Vinh hopes to attend medical school after graduation or seek employment in biomedical industry where he can apply his expertise to further the field of Biomedical Engineering. 